\title{
Revisiting the gas kinematics in SSA22 Lyman- $\alpha$ Blob 1 with radiative transfer modelling in a multiphase, clumpy medium
}

\author{
Zhihui Li ${ }^{\oplus, 1 \star}$ Charles C. Steidel ${ }^{\oplus, 1}$ Max Gronke ${ }^{\oplus 2,3} \dagger$ and Yuguang Chen ${ }^{\oplus 1}$ \\ ${ }^{1}$ Cahill Center for Astrophysics, California Institute of Technology, MC 249-17, 1200 East California Boulevard, Pasadena, CA 91125, USA \\ ${ }^{2}$ Department of Physics, University of California, Santa Barbara, CA 93106, USA \\ ${ }^{3}$ Department of Physics and Astronomy, Johns Hopkins University, Baltimore, MD 21218, USA
}

Accepted 2020 December 16. Received 2020 November 17; in original form 2020 August 24

\begin{abstract}
We present new observations of Lyman- $\alpha(\operatorname{Ly} \alpha)$ Blob 1 (LAB1) in the SSA22 protocluster region $(z=3.09)$ using the Keck Cosmic Web Imager and Keck Multi-object Spectrometer for Infrared Exploration. We have created a narrow-band Ly $\alpha$ image and identified several prominent features. By comparing the spatial distributions and intensities of $\mathrm{Ly} \alpha$ and $\mathrm{H} \beta$, we find that recombination of photo-ionized $\mathrm{H}$ I gas followed by resonant scattering is sufficient to explain all the observed $\mathrm{Ly} \alpha / \mathrm{H} \beta$ ratios. We further decode the spatially resolved Ly $\alpha$ profiles using both moment maps and radiative transfer modelling. By fitting a set of multiphase, 'clumpy' models to the observed Ly $\alpha$ profiles, we manage to reasonably constrain many parameters, namely the H I number density in the interclump medium (ICM), the cloud volume filling factor, the random velocity and outflow velocity of the clumps, the H I outflow velocity of the ICM, and the local systemic redshift. Our model has successfully reproduced the diverse Ly $\alpha$ morphologies, and the main results are: (1) the observed Ly $\alpha$ spectra require relatively few clumps per line of sight as they have significant fluxes at the line centre; (2) the velocity dispersion of the clumps yields a significant broadening of the spectra as observed; (3) the clump bulk outflow can also cause additional broadening if the H I in the ICM is optically thick; (4) and the $\mathrm{HI}$ in the ICM is responsible for the absorption feature close to the Ly $\alpha$ line centre.
\end{abstract}

Key words: galaxies: evolution-galaxies: high-redshift-intergalactic medium-galaxies: kinematics and dynamics.

\section{INTRODUCTION}

Lyman- $\alpha$ Blobs (LABs) - spatially extended (projected sizes $\gtrsim$ $100 \mathrm{kpc})$ gaseous nebulae at high redshift $(z \gtrsim 2)$ with immense Ly $\alpha$ luminosities $\left(L_{\mathrm{Ly} \alpha} \sim 10^{43-44} \mathrm{erg} \mathrm{s}^{-1}\right)$ - are among the most enigmatic and intriguing objects in the universe. To date, hundreds of LABs have been discovered (e.g. Francis et al. 1996; Fynbo, Møller \& Warren 1999; Keel et al. 1999; Steidel et al. 2000; Matsuda et al. 2004, 2011; Dey et al. 2005; Saito et al. 2006; Smith \& Jarvis 2007; Hennawi et al. 2009; Ouchi et al. 2009; Prescott, Dey \& Jannuzi 2009, 2012; Erb, Bogosavljević \& Steidel 2011; Cai et al. 2017), yet their physical origin remains murky. Many of the LABs have been found in overdense regions associated with massive protoclusters, which will presumably evolve into rich galaxy clusters observed today (e.g. Steidel et al. 1998; Prescott et al. 2008; Yang et al. 2009, 2010; Hine et al. 2016). Hence, the study of LABs may elucidate the formation process of massive galaxies and the mechanisms of concurrent feedback events.

What are the possible energy sources that power the observed Ly $\alpha$ emission of LABs? Thus far, numerous attempts have been made to answer this fundamental question, but a consensus is yet to be reached. Among many proposed scenarios, one of the most plausible Ly $\alpha$ production mechanisms is photo-ionization via

\footnotetext{
^E-mail: zhihui@caltech.edu

$\dagger$ Hubble fellow.
}

embedded energetic sources (e.g. starburst galaxies or active galactic nuclei, AGNs) followed by subsequent recombination (Haiman \& Rees 2001; Cantalupo et al. 2005, 2014). This scenario has been corroborated by the discovery of luminous galaxies and AGNs (Chapman et al. 2001; Dey et al. 2005; Geach et al. 2005, 2007, 2009; Colbert et al. 2006; Webb et al. 2009) inside some LABs via infrared (IR) and submillimetre (submm) observations. If the ionizing sources are starbursts, supernova-induced energetic winds may be triggered (Heckman, Armus \& Miley 1990; Taniguchi \& Shioya 2000; Taniguchi, Shioya \& Kakazu 2001; Mori, Umemura \& Ferrara 2004), producing outflowing superbubbles and additional Ly $\alpha$ emission via shock heating. Evidence for the existence of such 'superwinds' includes the observed double-peaked Ly $\alpha$ profiles (Ohyama et al. 2003) and bubble-like structures (Matsuda et al. 2004). Alternatively, Ly $\alpha$ emission can originate from cooling radiation via accretion of cold gas streams in dark matter haloes on to protogalaxies (Haiman, Spaans \& Quataert 2000; Fardal et al. 2001; Furlanetto et al. 2005; Dijkstra, Haiman \& Spaans 2006a, b; Scarlata et al. 2009; Goerdt et al. 2010; Faucher-Giguère et al. 2010; Rosdahl \& Blaizot 2012). This explanation is especially favoured for LABs with no or only weak associated energy sources identified even with deep multiwavelength observations (Nilsson et al. 2006; Smith \& Jarvis 2007; Saito et al. 2008; Smith et al. 2008). In either case, a substantial fraction of the Ly $\alpha$ photons will be resonantly scattered multiple times before escape (Steidel et al. 2010, 2011), although the 'cold accretion' scenario is supposed to induce a lower degree of polarization due to a lower chance of scattering from the 
inside out (Dijkstra \& Loeb 2009; Hayes, Scarlata \& Siana 2011; Trebitsch et al. 2016; Eide et al. 2018).

In this paper, we present new observations and analyses of one of the first LABs ever discovered, SSA22-Blob1 (LAB1, Steidel et al. 2000). LAB1 is one of the brightest and largest LABs discovered to date, with a Ly $\alpha$ luminosity of $\sim 1.1 \times 10^{44} \mathrm{erg} \mathrm{s}^{-1}$ (Weijmans et al. 2010) and a spatial extent of $\sim 100 \mathrm{kpc}$ (Matsuda et al. 2004). Since its discovery, LAB1 has been studied extensively, at wavelengths including X-ray (Geach et al. 2009), optical (Ohyama et al. 2003; Bower et al. 2004; Weijmans et al. 2010), IR (Uchimoto et al. 2008, 2012; Webb et al. 2009), and submm, Geach et al. 2005; Matsuda et al. 2007; Geach et al. 2014; Hine et al. 2016). Two Lyman-break galaxies (LBG), C11 and C15 (Steidel et al. 2000, 2003; Matsuda et al. 2004), and multiple dust-obscured star-forming galaxies (Geach et al. 2007, 2014, 2016) have been identified within LAB1. However, $\mathrm{X}$-ray observations yield non-detections, indicating the absence of (Compton-thin) AGNs (Geach et al. 2009).

To determine the principle energy source(s) powering LAB1, three main approaches have been adopted: the first is to infer the gas kinematics (e.g. inflows versus outflows) from the observed properties of $\mathrm{Ly} \alpha$ as well as other non-resonant emission lines (e.g. [O III], H $\alpha, \mathrm{H} \beta$ ). For example, Bower et al. (2004) and Weijmans et al. (2010) measured a velocity shear of the Ly $\alpha$ emission from C11 and C15 using integral-field spectroscopy, which suggests the presence of outflows. On the other hand, McLinden et al. (2013) reported a nearly zero velocity offset between $\mathrm{Ly} \alpha$ and [O III] in $\mathrm{C} 11$ and $\mathrm{C} 15$, which they interpreted as an absence of strong outflows. An alternative approach is to compare the available energy budget of possible energy sources with the observed Ly $\alpha$ emission. For example, Geach et al. (2016) deduced the IR luminosities and corresponding star formation rate $\left(\mathrm{SFR}, \sim 150 \mathrm{M}_{\odot} \mathrm{yr}^{-1}\right.$ ) of the embedded sources from their $850 \mu \mathrm{m}$ flux density measured with Atacama Large Interferometer Array (ALMA), and found that this energy budget is sufficient to power the observed Ly $\alpha$ luminosity. However, as it is difficult to independently constrain the fraction of Ly $\alpha$ photons that escape from the galaxy and scatter into our line of sight, additional energy sources (e.g. cold accretion) cannot be ruled out entirely (Geach et al. 2014; Hine et al. 2016). Thirdly, Hayes et al. (2011) and Beck et al. (2016) have measured polarized Ly $\alpha$ emission using polarimetric imaging. Although they claimed that this result should be strongly supportive of a 'central powering + scattering' model, Trebitsch et al. (2016) pointed out that the scattering inside the cold filaments in the "cold accretion' scenario could still account for the degree of polarization observed.

In this paper, we use an advanced kinematic approach to further test the feasibility of the 'central powering + scattering' scenario. The traditional kinematic approach - inferring the underlying gas velocity field from the observed peak shifts and line widths (e.g. McLinden et al. 2013) is worth scrutinizing, as resonant scattering may modify the line profiles in a very complex way. Instead, we model the Ly $\alpha$ profiles using Monte Carlo radiative transfer (MCRT). Due to its computationally expensive nature, Ly $\alpha$ MCRT modelling normally assumes a simple, idealized geometry, for example, a spherically symmetric expanding shell of $\mathrm{HI}$ gas surrounding a central Ly $\alpha$ emitting source (the 'shell model', Verhamme, Schaerer \& Maselli 2006; Dijkstra et al. 2006b). This simple model has successfully reproduced many observed Ly $\alpha$ spectra (e.g. Schaerer \& Verhamme 2008; Verhamme et al. 2008; Dessauges-Zavadsky et al. 2010; Vanzella et al. 2010; Gronke 2017), although it has also encountered some challenges for those with multiple peaks (Verhamme et al. 2008; Kulas et al. 2012; Rivera-Thorsen et al. 2017) or very large line widths (Hashimoto et al. 2015; Yang et al. 2016, 2017; Orlitová et al. 2018). Moreover, recent observations have shown increasing evidence that the circumgalactic medium (CGM), just like the interstellar medium (ISM), is multiphase and clumpy (e.g. the Ly $\alpha$ emission and metal absorption line observations of high-redshift quasars (Cantalupo et al. 2014; Hennawi et al. 2015)), which is further corroborated by simulations with increased spatial resolution (e.g. Hummels et al. 2019). Therefore, a more realistic model that accounts for the multiphase nature and clumpy geometry of H I gas is needed to properly characterize the radiative transfer processes of Ly $\alpha$ photons.

Up to now, this multiphase 'clumpy' model has been explored theoretically via both semi-analytical calculations (Neufeld 1991) and Monte Carlo simulations (Hansen \& Oh 2006; Dijkstra \& Kramer 2012; Laursen, Duval \& Östlin 2013; Gronke \& Dijkstra 2016). However, due to its complex and multivariate nature, the multiphase 'clumpy' model has not been widely used in fitting real Ly $\alpha$ spectra (albeit the first attempt made in Forero-Romero et al. 2018). In this work, we use the framework proposed by Gronke \& Dijkstra (2016) to model the spatially resolved Ly $\alpha$ spectra in LAB 1 .

In addition to the Ly $\alpha$ observations in the optical (rest-frame UV) using the Keck Cosmic Web Imager (KCWI, Martin et al. 2010; Morrissey et al. 2012), we have carried out near-IR (NIR, rest-frame optical) spectroscopic observations using the Keck Multiobject Spectrometer for Infrared Exploration (MOSFIRE, McLean et al. 2010, 2012). By comparing the spatial distribution of Ly $\alpha$, [O III], and $\mathrm{H} \beta$ emission and fitting $\mathrm{Ly} \alpha$ line profiles, we map the kinematic structure of $\mathrm{HI}$ in LAB1 and constrain its possible powering mechanism(s).

The structure of this paper is as follows. In Section 2, we describe our KCWI and MOSFIRE observations and data reduction procedures. In Section 3, we present our new observational results and analyses. In Section 4, we detail the methodology and present our results of radiative transfer modelling using the multiphase, clumpy model. In Section 5, we summarize and conclude. Throughout this paper, we adopt a flat $\Lambda$-cold dark matter cosmology with $\Omega_{\mathrm{m}}$ $=0.315, \Omega_{\Lambda}=0.685$, and $H_{0}=67.4 \mathrm{~km} \mathrm{~s}^{-1} \mathrm{Mpc}^{-1}$ (Planck Collaboration et al. 2020). We use the following vacuum wavelengths: $1215.67 \AA$ for $\mathrm{Ly} \alpha, 4862.683 \AA$ for $\mathrm{H} \beta$, and $4960.295 / 5008.240 \AA$ for [O III] from the Atomic Line List v2.04. ${ }^{1}$

\section{OBSERVATIONS AND DATA REDUCTION}

\subsection{KCWI observations}

The KCWI observations of LAB1 were carried out on the night of 2018 June 16, with a seeing of $\sim 1.0$ arcsec full width at halfmaximum (FWHM). We used the KCWI large slicer, which provides a contiguous field of view (FOV) of $20.4 \operatorname{arcsec}$ (slice length) $\times$ $33 \operatorname{arcsec}(24 \times 1.35$ arcsec slice width). With the blue medium $(\mathrm{BM})$ volume-phase holographic (VPH) grating set up for $\lambda_{\mathrm{c}}=4800 \AA$, the wavelength coverage is $\sim 4260-5330 \AA$, with spectral resolution $R \simeq 1800-2200$. The data were obtained as nine individual $1200 \mathrm{~s}$ exposures, with small telescope offsets in the direction perpendicular to slices applied between each, in an effort to recover some spatial resolution given the relatively large slice width. The total on-source exposure time was $3 \mathrm{~h}$.

Individual exposures were reduced using the KCWI Data Reduction Pipeline, ${ }^{2}$ which includes wavelength calibration, atmospheric

\footnotetext{
${ }^{1}$ http://www.pa.uky.edu/ peter/atomic/index.html

${ }^{2}$ https://github.com/Keck-DataReductionPipelines/KcwiDRP
} 

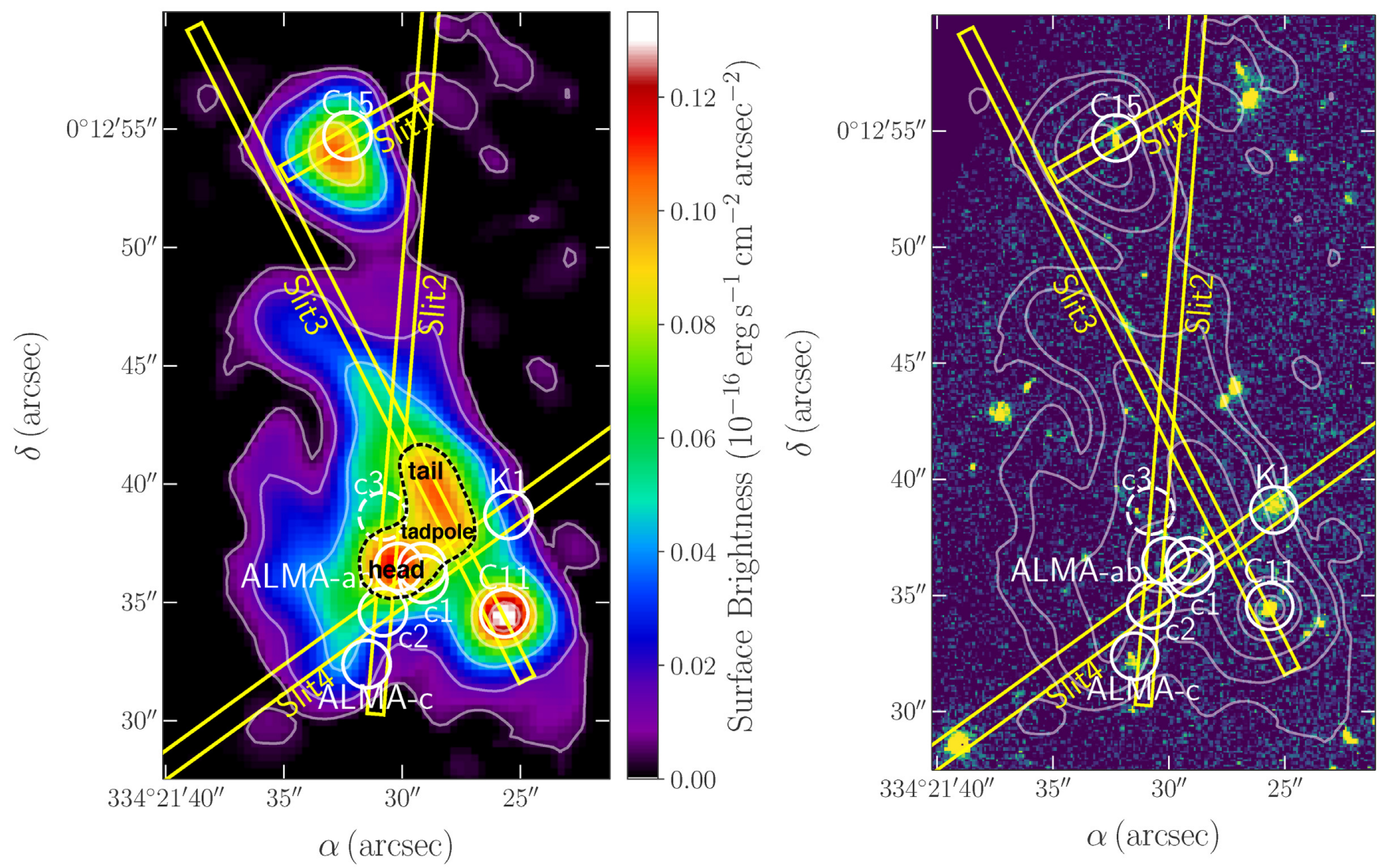

Figure 1. Ly $\alpha$ and continuum images of LAB1. Left: the narrow-band Ly $\alpha$ image, obtained by collapsing the original KCWI datacube over $4959-5009 \AA$, which contains the Ly $\alpha$ line (see Section 2). The UV continuum near the wavelength of Ly $\alpha$ has been subtracted. Right: the HST/STIS optical continuum image. The positions of four MOSFIRE slits (slit 1-4), two LBGs (C11 and C15), three submm sources (ALMA-a, b, and c), a $K$-band selected galaxy $(\mathrm{K} 1)$, and three $[\mathrm{O} \mathrm{III}] / \mathrm{Ly} \alpha$ serendipitous sources $\left(\mathrm{c}_{1}\right.$ to $\mathrm{c}_{3}$ ) have been marked on each image (see Table 2). The Ly $\alpha$ isophotes with levels of $\mathrm{SB}_{\mathrm{Ly} \alpha}$ $=[120,80,40,15,4] \times 10^{-19} \mathrm{erg} \mathrm{s}^{-1} \mathrm{~cm}^{-2} \operatorname{arcsec}^{-2}$ have also been overlaid. All images have been registered to the same world-coordinate system.

refraction correction, background subtraction, and flux calibration. The individual datacubes were then spatially re-sampled on to a uniform astrometric grid with 0.3 arcsec by 0.3 arcsec spaxels, with a sampling of $0.5 \AA \mathrm{pixel}^{-1}$ (4.75 pixels per spectral resolution element) along the wavelength axis, using a variant of the 'drizzle' algorithm (with a drizzle factor of 0.9 ) in the MONTAGE ${ }^{3}$ package. The re-sampled cubes were then combined into a final stacked cube by averaging with exposure time weighting. Owing to the coarser spatial sampling in the long dimension of the spatial cube, the PSF (point spread function) in the final datacube is elongated along the $\mathrm{N}-\mathrm{S}$ direction, with $\mathrm{FWHM} \simeq 0.96 \operatorname{arcsec} \times 1.44 \operatorname{arcsec}(X-$ and $Y$-directions, respectively).

The resampled final datacube covers a scientifically useful solid angle of 18.9 arcsec $\times 32.7$ arcsec on the sky, and a wavelength range (vacuum and heliocentric) of 4214-5243 A. A variance image with the same dimensions was created by propagating errors based on a noise model throughout the data reduction.

\subsection{MOSFIRE observations}

We observed selected regions of $\mathrm{LAB} 1$, chosen to include the highest Ly $\alpha$ SB areas as determined from a very deep narrowband Ly $\alpha$ image (see Steidel et al. 2011; Nestor et al. 2011) using

${ }^{3} \mathrm{http}: / /$ montage.ipac.caltech.edu
Table 1. MOSFIRE $K$-band observations of LAB1.

\begin{tabular}{lccccccc}
\hline $\begin{array}{l}\text { Name } \\
(1)\end{array}$ & $\begin{array}{c}\text { Width } \\
(2)\end{array}$ & $\begin{array}{c}R \\
(3)\end{array}$ & \multicolumn{1}{c}{$\begin{array}{c}\text { PA } \\
(4)\end{array}$} & $\begin{array}{c}\text { Exp } \\
(5)\end{array}$ & $\begin{array}{c}\text { Seeing } \\
(6)\end{array}$ & $\begin{array}{c}\text { Date of obs. } \\
(7)\end{array}$ & $\begin{array}{c}\text { Nod } \\
(8)\end{array}$ \\
\hline Slit 1 & 0.7 & 3660 & -68.0 & 4.0 & 0.50 & 2012 Sep15 & 3.0 \\
Slit 2 & 0.7 & 3660 & -3.5 & 1.5 & 0.43 & 2012 Jun30 & 3.0 \\
Slit 3 & 0.7 & 3660 & 27.0 & 1.5 & 0.52 & 2012 Sep13 & 3.0 \\
Slit 4 & 1.0 & 2560 & -54.0 & 2.5 & 0.53 & 2019 Jun15 & 15.0
\end{tabular}

Notes: The details of the MOSFIRE $K$-band observations of LAB1. The columns are: (1) slit name; (2) slit width $(\operatorname{arcsec})$; (3) resolving power $(\lambda / \Delta \lambda)$; (4) slit PA (degrees E of N); (5) exposure time in hours; (6) seeing FWHM (arcsec); (7) UT date of observation; and (8) nod amplitude between A and B positions (arcsec).

MOSFIRE (McLean et al. 2010, 2012; Steidel et al. 2014) on the Keck I telescope. Spectra in the near-IR $K$ band $(1.95-2.40 \mu \mathrm{m})$ were obtained using four different slitmasks, each of which included a slit passing through part of LAB1 with a different RA, Dec., and position angle (PA). The four slits are labelled as 'slit 1' through 'slit 4' in Fig. 1, and the observations are summarized in Table 1. Slits 1-3 were obtained using slits of width 0.7 arcsec, providing spectral resolving power of $R \simeq 3700$; slit 4 observations used a 1.0 arcsec wide slit, yielding $R \simeq 2600$. The observations were obtained during four different observing runs between 2012 June and 2019 November, under clear skies with seeing in the range $0.43-$ 0.53 arcsec, as summarized in Table 1 . 
The MOSFIRE $K$-band observations (slit 1 was also observed in $H$ band) were all obtained using an $\mathrm{ABAB}$ nod pattern along the slit direction with nod amplitude of 3 arcsec between positions A and B for slits 1,2 , and 3 , and 15 arcsec for slit 4 . Total integration times were 1.5-4.0 h, as listed in Table 1, composed of 30-80 individual $180 \mathrm{~s}$ exposures. The data for each observation sequence were reduced using the MOSFIRE data reduction pipeline ${ }^{4}$ to produce $2 \mathrm{D}$, rectified, background-subtracted vacuum wavelength calibrated spectrograms (see Steidel et al. 2014 for details). Observations obtained on different observing nights using the same slitmask were reduced independently; the $2 \mathrm{D}$ spectrograms were shifted into the heliocentric rest frame and combined with inverse variance weighting using tasks in the MOSPEC (Strom et al. 2017) analysis package.

\section{THE GAS KINEMATIC STRUCTURE OF LAB 1}

\subsection{Spatial distribution of $\mathrm{Ly} \alpha$ emission}

To get an overview of the Ly $\alpha$ surface brightness (SB) distribution in LAB1, we first generate a Ly $\alpha$ narrow-band image by optimally summing all the Ly $\alpha$ fluxes over the relevant wavelength range. Here we follow the 'matched filtering' procedures for creating a narrow-band image (Herenz, Hayes \& Scarlata 2020) using LSDCat (Herenz \& Wisotzki 2017). First, we apply spatial filtering to the continuum-subtracted KCWI datacube using a 2D Gaussian filter with a constant 1.2 arcsec FWHM, which equals the seeing PSF measured from a bright star in the SSA22 field. Secondly, we apply a 1D Gaussian spectral filter with FWHM $=1000 \mathrm{~km} \mathrm{~s}^{-1}$, which is the typical observed Ly $\alpha$ line width estimated via visual inspection. Thirdly, we use this filtered datacube to generate an signal-to-noise $(\mathrm{S} / \mathrm{N})$ cube. We can then choose appropriate $\mathrm{S} / \mathrm{N}$ thresholds for the filtered datacube to produce SB and moment maps (see Section 3.3).

In the left-hand panel of Fig. 1, we present our narrow-band Ly $\alpha$ image. It is constructed by summing over all the voxels of the filtered datacube with $\mathrm{S} / \mathrm{N} \geq 4$ over $4959-5009 \AA$, which should enclose all possible Ly $\alpha$ emission. To further examine whether the Ly $\alpha$ emission coincides with the identified sources, we also present the Hubble Space Telescope/Space Telescope Imaging Spectrograph (HST/STIS) optical continuum image of LAB $1 .^{5}$

We have also marked the positions of previously identified sources on each image as references. Among these sources, C11 and C15 are both LBGs (Steidel et al. 2000); ALMA-a, b, and c are three submm galaxies (Geach et al. 2016); $\mathrm{K} 1$ is a $K$-band selected galaxy (Kubo et al. 2015); $c_{1}$ and $c_{2}$ (the same as $\mathrm{S} 1$ in Geach et al. 2016) are two [O III] serendipitous sources; and $c_{3}$ is a Ly $\alpha$ serendipitous source at a lower redshift $(z=2.7542)$. The detailed information (especially spectroscopic redshifts, if available) of all the identified sources are presented in Table 2. The Ly $\alpha$ isophotes (contours with the same SB) with levels of $\mathrm{SB}_{\mathrm{Ly} \alpha}=[120,80,40,15,4] \times 10^{-19} \mathrm{erg} \mathrm{s}^{-1} \mathrm{~cm}^{-2} \operatorname{arcsec}^{-2}$ have also been overlaid on to each image.

Several prominent features are evident in Fig. 1: (1) In general, the regions with the highest SB are associated with identified sources (e.g. C11, C15, and ALMA-a), although the position of the maximum Ly $\alpha$ SB may be offset from the continuum source (e.g. C15); (2) an exception worth noting is a tadpole-shaped structure (marked in Fig. 1), which starts from the ALMA-ab sources, wriggles towards

\footnotetext{
${ }^{4}$ https://github.com/Keck-DataReductionPipelines/MosfireDRP

${ }^{5}$ The KCWI and HST/STIS images have been registered to the same worldcoordinate system using cross-correlation.
}

Table 2. Continuum sources identified in LAB1.

\begin{tabular}{lccccc}
\hline Name & RA (J2000) & Dec. (J2000) & $z_{\text {sys }}$ & \multicolumn{2}{c}{ Type References } \\
\hline C11 $^{a}$ & $22: 17: 25.70$ & $+00: 12: 34.7$ & $3.0980 \pm 0.0001$ & {$[\mathrm{O} \mathrm{III]}(1)$ and (2) } \\
C15 $^{a}$ & $22: 17: 26.15$ & $+00: 12: 54.7$ & $3.0975 \pm 0.0001$ & {$[\mathrm{O} \mathrm{III]}(1)$ and (2) } \\
ALMA-a & $22: 17: 25.94$ & $+00: 12: 36.6$ & $\ldots$ & $\ldots$ & $(3)$ \\
ALMA-b & $22: 17: 26.01$ & $+00: 12: 36.4$ & $\ldots$ & $\ldots$ & $(3)$ \\
ALMA-c & $22: 17: 26.11$ & $+00: 12: 32.4$ & $3.1000 \pm 0.0003$ & {$[\mathrm{C} \mathrm{III}]$} & $(5)$ \\
& $22: 17: 26.10$ & $+00: 12: 32.3$ & $3.0993 \pm 0.0004$ & {$[\mathrm{O} \mathrm{III]}$} & $(3)$ \\
K1 & $22: 17: 25.70$ & $+00: 12: 38.7$ & $3.1007 \pm 0.0002$ & {$[\mathrm{O} \mathrm{III]}$} & $(4)$ \\
$\mathrm{c}_{1}$ & $22: 17: 25.94$ & $+00: 12: 36.0$ & 3.0988 & {$[\mathrm{O} \mathrm{III]}$} & $(1)$ \\
$\mathrm{c}_{2} /$ S1 & $22: 17: 26.08$ & $+00: 12: 34.2$ & 3.0968 & {$[\mathrm{O} \mathrm{III]}$} & $(1)$ and (3) \\
$\mathrm{c}_{3}$ & $22: 17: 26.05$ & $+00: 12: 38.7$ & 2.7542 & Ly $\alpha$ & $(1)$ \\
\hline
\end{tabular}

Notes: ${ }^{a}$ Originally defined in Steidel et al. (2000).

References. (1) This work; (2) McLinden et al. (2013); (3) Geach et al. (2016); (4) Kubo et al. (2015); (5) Umehata et al. (2017).

the north-west first and then north-east. Interestingly, although the 'head' of the tadpole overlaps with ALMA-a, its 'tail' does not overlap with any source; and (3) the regions with identified continuum sources do not necessarily have significant Ly $\alpha$ emission (e.g. ALMA-c, $c_{2}, \mathrm{~K} 1$ ).

\subsection{Spatial distribution of [O III] and $\mathrm{H} \beta$ emission}

To test whether the extended Ly $\alpha$ emission is produced 'in situ' or 'ex situ' (the latter requires scattering), we further use MOSFIRE to map the spatial distribution of two other non-resonant lines, [O III] and $\mathrm{H} \beta$, and quantitatively compare them with $\operatorname{Ly} \alpha$ emission at the same spatial position. The positions of the four MOSFIRE slits are also shown in Fig. 1.

Theoretically, we consider two principal scenarios of Ly $\alpha$ production: (1) photo-ionization + recombination (e.g. due to star formation); and (2) collisional excitation + radiative de-excitation (e.g. due to cold accretion). For scenario 1, assuming case B recombination, we use the PYNEB package (Luridiana, Morisset \& Shaw 2015) to calculate $F_{\mathrm{Ly} \alpha} / F_{\mathrm{H} \beta}$ for $T_{\mathrm{HI}}(\mathrm{K}) \in\left[10^{3}, 10^{5}\right]$ and $n_{\mathrm{e}}\left(\mathrm{cm}^{-3}\right) \in[1$, $10^{4} \mathrm{~J}$, where $T_{\mathrm{HI}}$ and $n_{\mathrm{e}}$ are the kinetic temperature of the $\mathrm{HI}$ gas and electron number density, respectively. For scenario 2, assuming collisional ionization equilibrium, we use the Chiant i Py package (Dere et al. 1997; Dere 2013; Dere et al. 2019) to calculate $F_{\mathrm{Ly} \alpha} / F_{\mathrm{H} \beta}$ for the same ranges of $T_{\mathrm{HI}}$ and $n_{\mathrm{e}}$ as above. The derived $F_{\mathrm{Ly} \alpha} / F_{\mathrm{H} \beta}$ as a function of $T_{\mathrm{HI}}$ for both scenarios are shown in Fig. 2. It can be seen that as $T_{\mathrm{HI}}$ increases, the predicted $F_{\mathrm{Ly} \alpha} / F_{\mathrm{H} \beta}$ is roughly constant for scenario 1 , but decreases for scenario 2 , as $\operatorname{Ly} \alpha$ emissivity drops more quickly than $\mathrm{H} \beta$.

Now we compare our spectroscopic data to the theoretical predictions above. For each MOSFIRE slit in Fig. 1, we construct a corresponding pseudo-slit to extract a $2 \mathrm{D}$ spectrum from the $3 \mathrm{D}$ KCWI datacube via a 3D datacube visualization tool QFitsView (Davies et al. 2010; Ott 2012). We then integrate the flux density in the wavelength dimension for each line and convert it to an SB accounting for the slit width.

In Fig. 3, we show the line SB for $\mathrm{Ly} \alpha$, [O III] and $\mathrm{H} \beta$ along each slit. Evidently, Ly $\alpha$ is not necessarily cospatial with [O III] or $\mathrm{H} \beta$, and is usually more extended along the slit. We further calculate $F_{[\mathrm{OIII}]} / F_{\mathrm{H} \beta}$ (shown in red numbers) by integrating $\mathrm{SB}_{\mathrm{H} \beta}$ and $\mathrm{SB}_{[\mathrm{O} \mathrm{III]}}$ along the slits for each identified source. We also calculate $F_{\mathrm{Ly} \alpha} / F_{\mathrm{H} \beta}$ in two ways, where $F_{\mathrm{Ly} \alpha}$ is calculated either by integrating $\mathrm{SB}_{\mathrm{Ly} \alpha}$ over the same region as $\mathrm{H} \beta$ and [O III] (the 'restricted' region, as indicated by solid arrows), or by integrating over the full extent of Ly $\alpha$ (the 'extended' region, as indicated by dashed arrows). The 


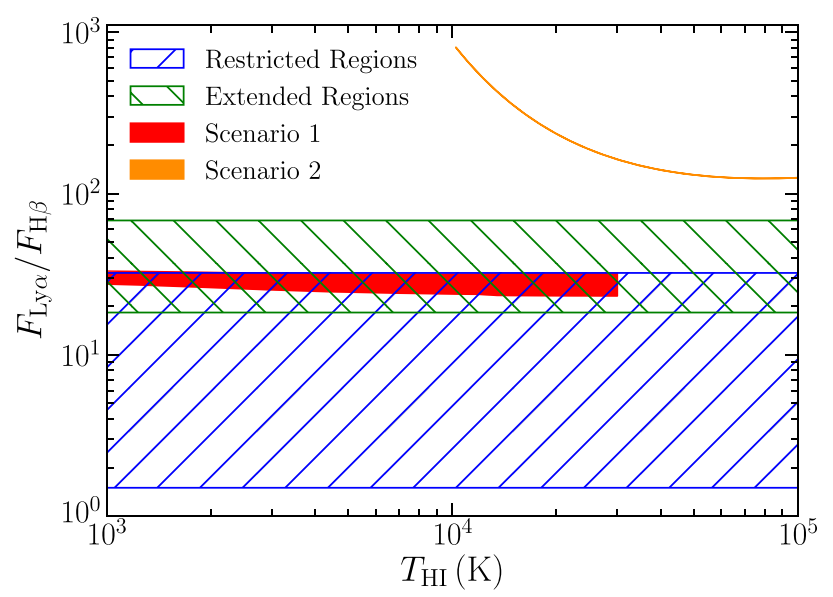

Figure 2. $F_{\mathrm{Ly} \alpha} / F_{\mathrm{H} \beta}$ as a function of the $\mathrm{HI}$ gas temperature $T_{\mathrm{HI}}$ in the photoionization (red patch) and collisional excitation (orange curve) scenarios. Also shown are the ranges of $F_{\mathrm{Ly} \alpha} / F_{\mathrm{H} \beta}$ measured along four MOSFIRE slits for both the restricted regions (shaded in blue slashes) and extended regions (shaded in green slashes) (see Section 3.2 for the definitions of restricted and extended regions).

results are shown next to the arrows (red for $F_{[\mathrm{O} \mathrm{IIII}} / F_{\mathrm{H} \beta}$ and green for $\left.F_{\mathrm{Ly} \alpha} / F_{\mathrm{H} \beta}\right)$.

The results in Figs 2 and 3 show that: (1) for slit 1 and $2, F_{\mathrm{Ly} \alpha} / F_{\mathrm{H} \beta}$ are always smaller than the predicted value of scenario 1 . Considering that $\mathrm{Ly} \alpha$ is subject to heavier dust extinction ${ }^{6}$ than $\mathrm{H} \beta$, this result suggests that scenario 1 itself is sufficient to explain the observed $F_{\mathrm{Ly} \alpha} / F_{\mathrm{H} \beta} ;$ (2) for slits 3 and 4 , we do see $F_{\mathrm{Ly} \alpha} / F_{\mathrm{H} \beta}$ ratios $(\sim 50-$ 70 , see Table 3 ) higher than that predicted by scenario 1 , but still far lower than those predicted by scenario 2 (especially in the $T_{\mathrm{HI}}$ $\geq 10^{4} \mathrm{~K}$ region, where both $\mathrm{Ly} \alpha$ and $\mathrm{H} \beta$ have been sufficiently excited). Simply scenario 1 and resonant scattering are sufficient to explain all the observed line ratios. Furthermore, we do not see a significant number of $\operatorname{Ly} \alpha$ profiles that have a blue dominant peak (signature of cold accretion, see e.g. Zheng \& Miralda-Escudé 2002; Dijkstra et al. 2006b; Faucher-Giguère et al. 2010) in these regions. Therefore, it is highly likely that photo-ionization + recombination is the main source of Ly $\alpha$ photons, and resonant scattering (as indicated by significant polarization detections from Hayes et al. 2011; Beck et al. 2016) has substantially altered their spatial and kinematic distribution.

\subsection{Profiles of $\mathrm{Ly} \alpha$ emission}

In this section, we investigate the variations of spatially-resolved Ly $\alpha$ profiles in major emitting regions. Before proceeding, we first use [O III] to determine the systemic redshifts of three associated sources: $\mathrm{C} 11, \mathrm{C} 15$, and $\mathrm{c}_{1}$. Single Gaussian fits to the [O III] line profiles (see Appendix A for details) yield redshifts (after heliocentric corrections) $z(\mathrm{C} 11)=3.0980, z(\mathrm{C} 15)=3.0975,{ }^{7}$ and $z\left(\mathrm{c}_{1}\right)=3.0988$, which we adopt as fiducial redshifts in the following analysis.

${ }^{6}$ Additionally, the scattering of $\operatorname{Ly} \alpha$ photons out of the line of sight can also reduce the observed $F_{\mathrm{Ly} \alpha} / F_{\mathrm{H} \beta}$ ratio.

${ }^{7}$ Compared to McLinden et al. (2013), our measurements for $z(\mathrm{C} 15)$ are consistent but our $z(\mathrm{C} 11)$ are slightly different. This may be due to: (1) the asymmetric nature of the [O III] profile of $\mathrm{C} 11$; and (2) the misalignment between the MOSFIRE slit and the galaxy continuum emission.
We then visualize the spatial variations of Ly $\alpha$ peak position $\left(v_{\mathrm{Ly} \alpha}\right)$ and line width $\left(\sigma_{\text {Ly } \alpha}\right)$ by making moment maps. The first and second flux-weighted moments are defined as:

$v_{\text {Ly } \alpha, x y}=\frac{\sum_{k} v_{x y k} I_{x y k}}{\sum_{k} I_{x y k}}$

$\sigma_{\mathrm{Ly} \alpha, x y}=\sqrt{\frac{\sum_{k}\left(v_{x y k}-v_{\mathrm{Ly} \alpha, x y}\right)^{2} I_{x y k}}{\sum_{k} I_{x y k}}}$

where $I_{x y k}$ and $v_{x y k}$ are the flux density and velocity (relative to a fiducial redshift) of the $k$ th wavelength layer at position $(x, y)$. In our moment analysis we fix the fiducial redshift of LAB1 at $z=3.1$, and all the summations are carried out over 4959-5009 $\AA$. Before applying equations (1) and (2), we filter out all the voxels with $\mathrm{S} / \mathrm{N}$ $<6$ (for $v_{\mathrm{Ly} \alpha}$ ) or 4 (for $\sigma_{\mathrm{Ly} \alpha}$ ). ${ }^{8}$ The $\sigma_{\mathrm{Ly} \alpha}$ map has been further corrected for the KCWI instrumental line spread function (LSF), $\sigma$ $=65 \mathrm{~km} \mathrm{~s}^{-1}$.

The resulting moment maps are shown in Fig. 4. The two major Ly $\alpha$ emitting regions have been delineated by rectangular boxes. We zoom in on these two regions in separate panels. By adjusting the dynamic range, we are able to discern more subtle structures, discussed in the following sections (Sections 3.3.1 and 3.3.2).

\subsubsection{Northern region}

There is only one identified source (C15) in the northern Ly $\alpha$ emitting region. We first use a large aperture to measure the global properties of LBG C15. The global line widths of Ly $\alpha$ and [O III] of C15 are 250 and $64 \mathrm{~km} \mathrm{~s}^{-1}$ (corrected for LSF, $\sigma=65 \mathrm{~km} \mathrm{~s}^{-1}$ for KCWI and $35 \mathrm{~km} \mathrm{~s}^{-1}$ for MOSFIRE). The global velocity offset between Ly $\alpha$ and [O III] is $\Delta v_{\mathrm{Ly} \alpha}=-22 \mathrm{~km} \mathrm{~s}^{-1}$, although it varies at different locations. This $\Delta v_{\text {Ly } \alpha}$ is significantly smaller than the velocity offsets observed in LBGs (Steidel et al. 2010) and LAEs (McLinden et al. 2011), both of which are $\gtrsim 300 \mathrm{~km} \mathrm{~s}^{-1}$ and are interpreted as signs of outflows. Therefore, it is tempting to conclude that this region should be lack of significant outflows. However, as we will show in Section 4.2, our multiphase, clumpy model predicts that significant outflow velocities can still be present in profiles with $\Delta v_{\text {Ly } \alpha} \simeq 0$ (e.g. in our spectra 1 and 2).

Most Ly $\alpha$ profiles in the northern region are considerably asymmetric and consist of a 'main peak' and a 'red bump' (see spectrum 1 in Fig. 5 as an example). Moreover, the main peak is redshifted towards the eastern region, and blueshifted towards the west. The largest $v_{\text {Ly } \alpha}$ can be up to $\sim 500 \mathrm{~km} \mathrm{~s}^{-1}$, which explains the evident east-west $v_{\text {Ly } \alpha}$ gradient in Fig. 4 . This shear in $v_{\text {Ly } \alpha}$ appears to be perpendicular to the major axis of $\mathrm{C} 15$, which is consistent with the suggestion by Weijmans et al. (2010) that outflow or rotation is indicated.

As for $\sigma_{\text {Ly } \alpha}$, its largest value $\left(\sim 400 \mathrm{~km} \mathrm{~s}^{-1}\right)$ is located slightly north-east of $\mathrm{C} 15$, beyond which $\sigma_{\text {Ly } \alpha}$ gradually decreases moving away from $\mathrm{C} 15$. In general, the $\sigma_{\mathrm{Ly} \alpha}$ values in the northern region are much larger than the global $\sigma_{[\mathrm{O} \mathrm{II}]}$. This is unexpected if one were to assume that both $\mathrm{Ly} \alpha$ and [O III] photons are emitted by the same sources, unless the kinematics of Ly $\alpha$ have been altered by radiative transfer effects. We attempt to explain the broadening of Ly $\alpha$ in Section 4.

${ }^{8}$ Our experiments show that these choices maximize the inclusion of real signal without introducing spurious detections. 
Table 3. Ly $\alpha$, [O III] and $\mathrm{H} \beta$ fluxes and line ratios measured along four MOSFIRE slits.

\begin{tabular}{|c|c|c|c|c|c|c|c|}
\hline \multirow[b]{2}{*}{$\begin{array}{l}\text { Slit no. } \\
\text { (1) }\end{array}$} & \multicolumn{5}{|c|}{ Restricted regions } & \multicolumn{2}{|c|}{ Extended regions } \\
\hline & $\begin{array}{l}\text { Ly } \alpha \\
\text { (2) }\end{array}$ & $\begin{array}{c}{[\mathrm{O} \text { III] }} \\
(3)\end{array}$ & $\begin{array}{l}\mathrm{H} \beta \\
\text { (4) }\end{array}$ & $\begin{array}{c}\mathrm{Ly} \alpha / \mathrm{H} \beta \\
\quad(5)\end{array}$ & $\begin{array}{c}{[\mathrm{O} \mathrm{III}] / \mathrm{H} \beta} \\
\quad(6)\end{array}$ & $\begin{array}{l}\operatorname{Ly} \alpha \\
\text { (7) }\end{array}$ & $\begin{array}{c}\mathrm{Ly} \alpha / \mathrm{H} \beta \\
\quad(8)\end{array}$ \\
\hline 1 & 4.8 & 2.3 & 0.4 & 9.6 & 5.8 & 9.3 & 18.3 \\
\hline 2 & 1.3 & 1.5 & 0.7 & 1.5 & 2.3 & 20.9 & 24.4 \\
\hline 3 & 7.5 & 1.2 & 0.3 & 17.3 & 3.5 & 29.3 & 68.1 \\
\hline 4 & $9.2 / 4.3$ & $0.6 / 1.1$ & $0.3 / 0.2$ & $32.2 / 23.4$ & $2.0 / 5.2$ & 24.3 & 51.8 \\
\hline
\end{tabular}

Notes: $\mathrm{Ly} \alpha,[\mathrm{O} \mathrm{III}]$, and $\mathrm{H} \beta$ fluxes measured by integrating along the four MOSFIRE slits (see Fig. 1 and Table 1 for details) and the corresponding extracted pseudo-slits from the KCWI datacube and line ratios $(\mathrm{Ly} \alpha / \mathrm{H} \beta$ and $[\mathrm{O}$ III $] / \mathrm{H} \beta)$. The columns are: (1) the slit number (as marked in Fig. 1); (2) the Ly $\alpha$ flux of the restricted regions (where $F_{\text {Ly } \alpha}$ is calculated over the same region as $\mathrm{H} \beta$ and [O III] , as indicated by solid arrows in Fig. 3); (3) the [O III] flux; (4) the $\mathrm{H} \beta$ flux; (5) the $\mathrm{Ly} \alpha / \mathrm{H} \beta$ ratio; (6) the $[\mathrm{O}$ III] $/ \mathrm{H} \beta$ ratio; (7) and (8) same as (2) and(5), but for extended regions (where $F_{\mathrm{Ly} \alpha}$ is calculated over the full extent of $\mathrm{Ly} \alpha$, as indicated by dashed arrows in Fig. 3). All fluxes are in units of $10^{-17} \mathrm{erg} \mathrm{s}^{-1} \mathrm{~cm}^{-2}$.

\subsubsection{Southern region}

Multiple discrete continuum sources have been identified within the southern portion of LAB1, including the LBG C11, three ALMA submm sources, and several very faint objects with spectroscopic confirmation $\left(\mathrm{K} 1, \mathrm{c}_{1}, \mathrm{c}_{2}\right.$, and $\left.\mathrm{c}_{3}\right)$. We first use a large aperture to measure the global properties of LBG C11. The LSF-corrected global line widths of Ly $\alpha$ and [O III] of C11 are 178 and $78 \mathrm{~km} \mathrm{~s}^{-1}$. The global velocity offset between Ly $\alpha$ and [O III] is $\Delta v_{\mathrm{Ly} \alpha}=+175 \mathrm{~km} \mathrm{~s}^{-1}$ (i.e. redshifted with respect to systemic), and $-197 \mathrm{~km} \mathrm{~s}^{-1}$ between the $\mathrm{Si}$ II 1526 absorption line (from Low Resolution Imaging Spectrometer (LRIS) observations) and Ly $\alpha$. Similar velocity offsets between Ly $\alpha$ and interstellar absorption features are commonly observed in 'down the barrel' spectra of LBGs, and are generally interpreted as signatures of outflow (Steidel et al. 2010). However, they are inconsistent with the non-detection of s significant offset between Ly $\alpha$ and [O III] by McLinden et al. (2011). This may be due to the high asymmetry of the [O III] profile of $\mathrm{C} 11$.

Most Ly $\alpha$ profiles from spatial locations near C11 exhibit double peaks - a red dominant peak + a blue 'bump' (see spectrum 10 in Fig. 5 as an example). The position of the red dominant peak tends to move towards more blueshifted velocities along the north-westsoutheast direction. The largest $v_{\text {Ly } \alpha}$ is $\sim 300 \mathrm{~km} \mathrm{~s}^{-1}$, which gives rise to the $v_{\text {Ly } \alpha}$ north-west-southeast gradient in Fig. 4 . This shear in $v_{\text {Ly } \alpha}$ appears to be parallel to the major axis of $\mathrm{C} 11$, consistent with Weijmans et al. (2010).

As for $\sigma_{\text {Ly } \alpha}$, its largest value $\left(\sim 500 \mathrm{~km} \mathrm{~s}^{-1}\right)$ is located in the southwest corner, while the majority of the spectra around $\mathrm{C} 11$ have a rather homogeneous $\sigma_{\mathrm{Ly} \alpha} \sim 400 \mathrm{~km} \mathrm{~s}^{-1}$. Again, these values are much larger than $\sigma_{[\mathrm{O} I \mathrm{III}]}$.

The Ly $\alpha$ profiles near the ALMA sources are more complex - most of them are very broad, highly asymmetric, and have multiple peaks. Some of the profiles (e.g. the northeast corner) are even dominated by a 'blue peak', as shown in spectrum 4 in Fig. 5.

On the $v_{\text {Ly } \alpha}$ map, there is an alternate pattern of positive and negative $v_{\text {Ly } \alpha}$ from the east to the west. Yet again, we see a similar coherent velocity structure that coincides with the high SB 'tadpole' structure (see Section 3.1). This structure is also seen on the $\sigma_{\text {Ly } \alpha}$ map, but with a slightly different trend - starting from the south, first going towards northeast, and then turning north-west. The largest $\sigma_{\text {Ly } \alpha}$ values $\left(\sim 500 \mathrm{~km} \mathrm{~s}^{-1}\right)$ still overlap with ALMA-a, which indicates that the ALMA source may be responsible for the Ly $\alpha$ line broadening (e.g. via starburst-driven outflows).

Our $v_{\operatorname{Ly} \alpha}$ and $\sigma_{\operatorname{Ly} \alpha}$ maps are qualitatively similar to the ones presented in a recent work by Herenz et al. (2020), albeit with slight differences in the extent of the Ly $\alpha$ emitting regions and the number of spaxels included, due to different FOVs of instruments and S/N threshold choices. The alternate pattern of positive and negative $v_{\mathrm{Ly} \alpha}$ is consistent with the left-hand panel in their fig. 7, and the large $\sigma_{\text {Ly } \alpha}$ values near ALMA-ab sources are consistent with the righthand panel in their fig. 7 .

\section{RADIATIVE TRANSFER MODELLING USING THE MULTIPHASE CLUMPY MODEL}

\subsection{Methodology}

Although the moment map analysis above provides a cursory overview of the apparent gas velocity field, it is purely phenomenological and could even be misleading, in the sense that if radiative transfer effects dominate, the observed $v_{\text {Ly } \alpha}$ would not necessarily be linked directly to the local gas kinematics. To gain more physical insight and to account for the possibly important radiative transfer effects, we generated a series of model spectra using MCRT and fit them to the observed Ly $\alpha$ spectra at different positions in LAB1.

Our first attempt was to fit the Ly $\alpha$ profiles using the widely used 'shell model' (Verhamme et al. 2006; Dijkstra et al. 2006b). However, as most line profiles are fairly broad and multipeaked with significant flux close to line centrer, the fits either fail to reproduce the major features or have inexplicably large intrinsic line widths (see e.g. Orlitová et al. 2018). Therefore, we adopt a more sophisticated and physically realistic multiphase 'clumpy model' instead. As described in Gronke \& Dijkstra (2016), the geometric setup of this 'clumpy model' is a number of spherical H I clumps moving within a hot $(T \sim$ $10^{5-7} \mathrm{~K}$ ), ionized interclump medium (ICM, see also Laursen et al. 2013). This model predicts the Ly $\alpha$ spectra produced by a central Ly $\alpha$ emitting source, accounting for the scattering by $\mathrm{HI}$ (both in the clumps and the ICM). ${ }^{9}$ It has 14 parameters in total (see the detailed formulation in Gronke \& Dijkstra 2016), among which the most important ones are the cloud covering factor $\left(f_{\mathrm{cl}}\right)$ that describes the mean number of clumps per line of sight from the centre to the boundary of the simulation sphere, the HI number density in the ICM $\left(n_{\mathrm{HI}, \mathrm{ICM}}\right)$, and kinematic parameters of the clumps and ICM. Specifically, the clump motion is assumed to be a superposition of an isotropic Gaussian random motion (characterized by $\sigma_{\mathrm{cl}}$, the

\footnotetext{
${ }^{9}$ As we will show below, the scattering process washes out the information about the Ly $\alpha$ emitting source, that is, the initial spatial or spectral shape of the source does not significantly affect the emergent spectra.
} 

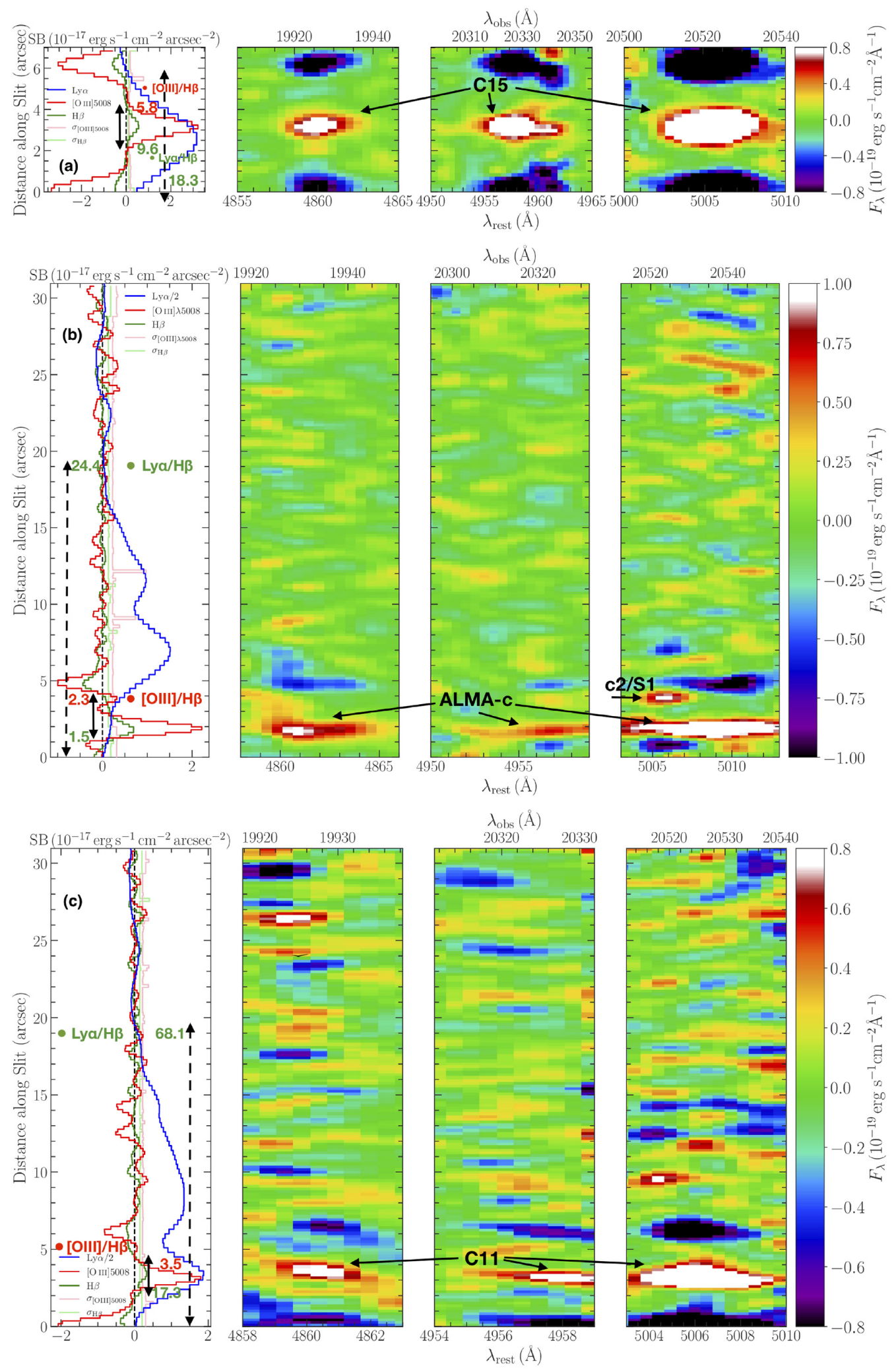

Figure 3. SB distributions of $\mathrm{Ly} \alpha$ (blue), [O III] (red), and $\mathrm{H} \beta$ (green) along four MOSFIRE slits (the leftmost panels, (a)-(d) correspond to slits 1-4, respectively). For reference, the smoothed $2 \mathrm{D}$ spectra of $\mathrm{H} \beta$ (the second column) and [O III] (the third and fourth columns, for 4960 and $5008 \AA$, respectively) are also shown. Both the observed wavelengths $\left(\lambda_{\text {obs }}\right)$ and the rest-frame wavelengths $\left(\lambda_{\text {rest }}\right.$, assuming $\left.z=3.1000\right)$ are shown. The positions of known sources are indicated with black arrows. We calculate $F_{[\mathrm{O}}{ }_{\mathrm{III}]} / F_{\mathrm{H} \beta}$ (shown in red numbers) by integrating $\mathrm{SB}_{\mathrm{H} \beta}$ and $\mathrm{SB}_{[\mathrm{O}}$ III] along the slits for each identified source. We also calculate $F_{\mathrm{Ly} \alpha} / F_{\mathrm{H} \beta}$ (shown in green numbers) in two ways, where $F_{\mathrm{Ly} \alpha}$ is calculated either by integrating $\mathrm{SB}_{\mathrm{Ly} \alpha}$ over the same region as $\mathrm{H} \beta$ and [O III] (as indicated by solid arrows), or by integrating over the full extent of Ly $\alpha$ (as indicated by dashed arrows). Note that the blue regions in the 2D spectra are negative images due to dithering. 


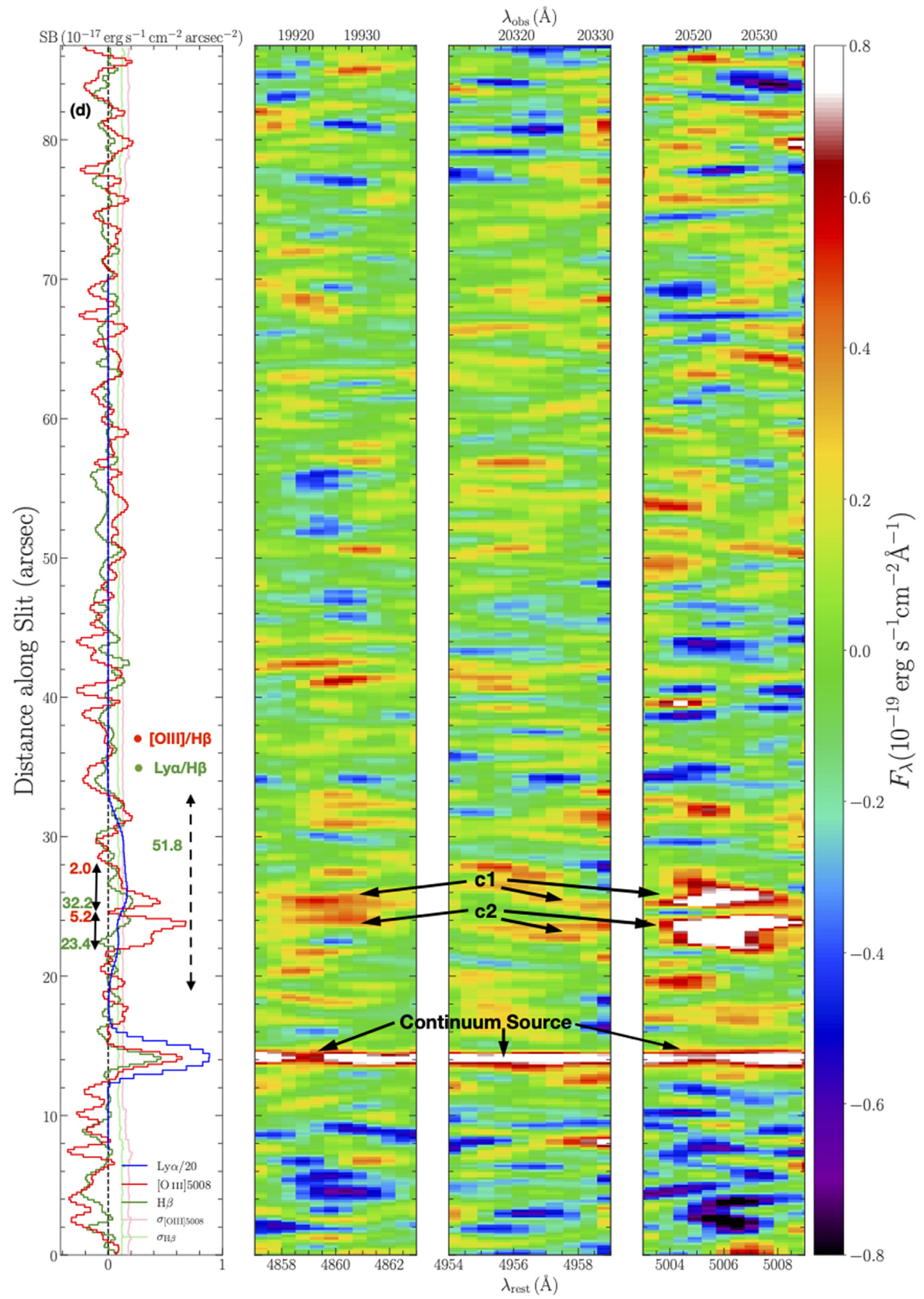

Figure 3. continued.

velocity dispersion of the clumps) and a radial uniform outflow with a constant velocity $v_{\mathrm{cl}}$. In addition, we consider an outflow velocity of the low density $\mathrm{H}$ I in the ICM, $v_{\mathrm{ICM}}$, and a post-processed parameter, the velocity shift with respect to $z=3.1000, \Delta v$. This $\Delta v$ parameter represents the best-fitting systemic redshift of the
Ly $\alpha$ source function relative to $z=3.1000$ (the initial guess for the systemic redshift).

Note that Ly $\alpha$ radiative transfer in such a multiphase medium exhibits two characteristic regimes defined by the values of $f_{\mathrm{cl}}$. If $f_{\mathrm{cl}}$ is (much) greater than a critical value $f_{\mathrm{cl} \text {, crit }}$ (which is a 

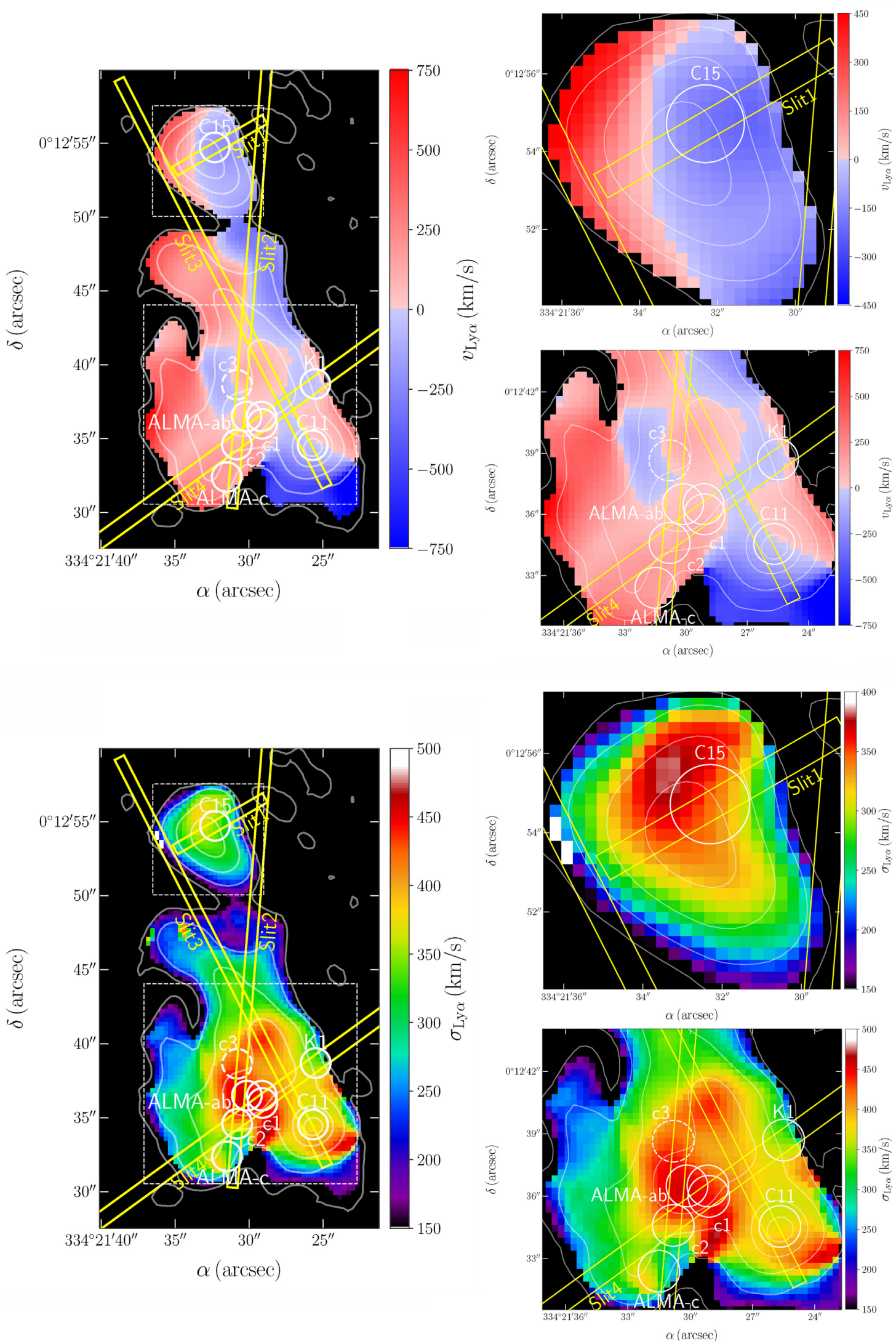

Figure 4. The first $\left(v_{\mathrm{Ly} \alpha}\right)$ and second $\left(\sigma_{\mathrm{Ly} \alpha}\right)$ moment maps of LAB1. The two major Ly $\alpha$ emitting regions have been delineated by rectangular boxes (dashed white lines), and their zooming-in views are shown in the right-hand panels. For the northern region, the colour bar limits have been adjusted accordingly to account for the smaller value range. The positions of the identified continuum sources are indicated by circles with labels. The Ly $\alpha$ SB isophotes (solid white lines) with levels of $\mathrm{SB}_{\mathrm{Ly} \alpha}=[120,80,40,15,4] \times 10^{-19} \mathrm{erg} \mathrm{s}^{-1} \mathrm{~cm}^{-2} \operatorname{arcsec}^{-2}$ have also been overlaid on to each image for visual reference. 

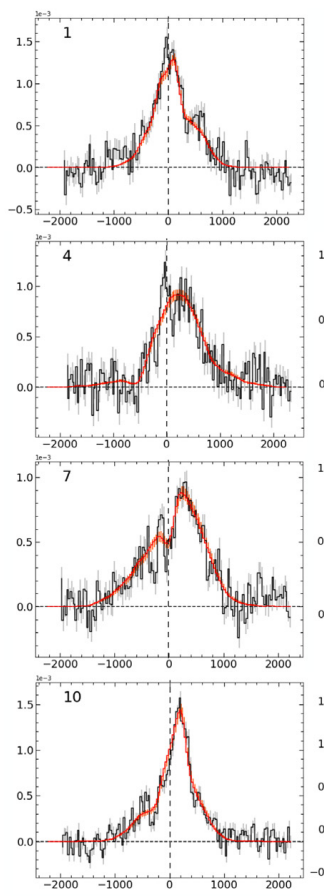
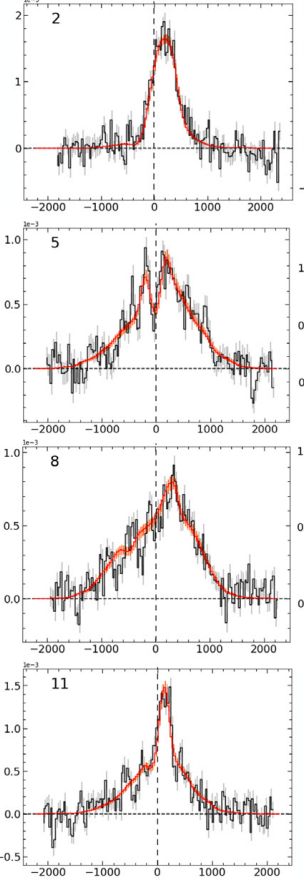
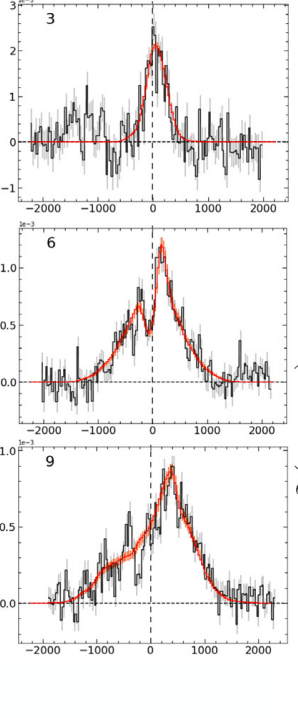

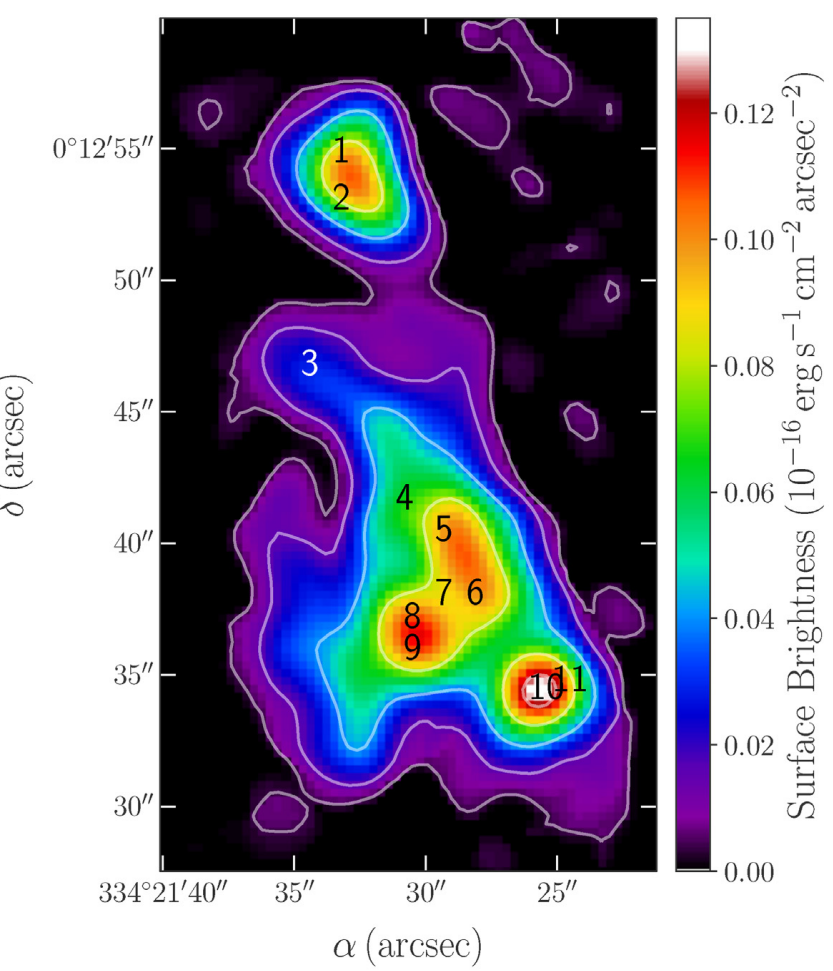

Figure 5. Eleven representative continuum-subtracted, spatially resolved Ly $\alpha$ profiles from the high SB regions in LAB1. All the spectra have been smoothed by a 3 pixel $\times 3$ pixel boxcar $(0.9$ arcsec $)$ spatially and Gaussian smoothed $(\sigma=0.5 \AA)$ in the wavelength dimension. The multiphase clumpy model best fits (red, with orange $1 \sigma$ Poisson errors) and the observed Ly $\alpha$ profiles (black, with grey $1 \sigma$ error bars) have both been normalized. The observed Ly $\alpha$ spectra have also been shifted by $-\Delta v$ to their local systemic redshifts. For each subpanel, the $x$-axis is the velocity (in $\mathrm{km} \mathrm{s}^{-1}$ ) with respect to the local systemic redshift, and the $y$-axis is the normalized line flux. The spectrum number of each spectrum has been marked on the SB map (the right-hand panel). For visual reference, the horizontal and vertical black dashed lines in each subpanel indicate zero flux level and zero velocity with respect to the local systemic redshift, respectively.

function of other model parameters, such as the kinematics and $\mathrm{HI}$ column density of the clumps), the photons would escape as if the medium is homogeneous and the emergent spectra are similar to the ones predicted by the aforementioned 'shell model' (Gronke et al. 2017). Otherwise, for fewer clumps per line-of-sight, the photons preferentially travel in the ionized ICM and escape closer to the line centre of Ly $\alpha$. As most of our observed Ly $\alpha$ spectra have considerable flux near the line centre, we expect $f_{\mathrm{cl}} \lesssim f_{\mathrm{cl} \text {, crit }}$ in our cases, and will focus on that regime. ${ }^{10}$

Based on these considerations, we further construct a 5D hypercubic grid by varying five crucial physical parameters: $\left[\log n_{\mathrm{HI}, \mathrm{ICM}}, F_{\mathrm{V}}, \sigma_{\mathrm{cl}}, v_{\mathrm{cl}}, v_{\mathrm{ICM}}\right] .{ }^{11}$ The prior ranges of $\log n_{\mathrm{HI}, \mathrm{ICM}}\left(\mathrm{cm}^{-3}\right), F_{\mathrm{V}}$, and $\left[\sigma_{\mathrm{cl}}, v_{\mathrm{cl}}, v_{\mathrm{ICM}}\right]\left(\mathrm{km} \mathrm{s}^{-1}\right)$ are [-8, -6$]$, [0.1, $0.6]$, and $[0,800]$ (with spacings of $0.4,0.1$, and 100 ), respectively. We fix the subdominant parameters, such as the ICM temperature $T_{\text {ICM }}$ to $10^{6} \mathrm{~K}$, and the clump column density to $10^{17} \mathrm{~cm}^{-2}$ in order to reduce the dimensionality of the parameter space. ${ }^{12}$

\footnotetext{
${ }^{10}$ Note that the model spectra are sensitive to $\sim f_{\mathrm{cl}} / f_{\mathrm{cl} \text {, crit }}$ but not to certain individual model parameters, such as the $\mathrm{H}$ I column density or the shape of the clumps (Hansen \& Oh 2006).

${ }^{11}$ For convenience, we vary $F_{\mathrm{V}}$ rather than $f_{\mathrm{cl}}$ when generating clumps, but they are directly related via $f_{\mathrm{cl}}=3 r_{\mathrm{gal}} / 4 r_{\mathrm{cl}} F_{\mathrm{V}}$, where $r_{\mathrm{gal}}=5 \mathrm{kpc}$ is the radius of the simulation sphere and $r_{\mathrm{cl}}=50 \mathrm{pc}$ is the clump radius (hence $f_{\mathrm{cl}}=75 F_{\mathrm{V}}$ in our case) .

${ }^{12}$ Here, we modelled each observed spectrum with a set of parameters of the scattering medium independently, whereas in reality the $\mathrm{Ly} \alpha$ photons are likely to be scattered by a common medium with spatially varying parameters.
}

Such a configuration amounts to 26244 models in total. Each model is calculated via radiative transfer using 10000 Ly $\alpha$ photon packages generated from a Gaussian intrinsic spectrum $N\left(0, \sigma_{\mathrm{cl}}^{2}\right)$, where $\sigma_{\mathrm{cl}}=12.85 \mathrm{~km} \mathrm{~s}^{-1}$ is the canonical thermal velocity dispersion of $T=10^{4} \mathrm{~K}$ gas. ${ }^{13}$ The sixth parameter, $\Delta v$, is varied continuously in post-processing. To properly explore the multimodal posterior of the parameters, we further use a PYTHON nested sampling package dynesty (Skilling 2004, 2006; Speagle 2020) to fit the Ly $\alpha$ spectra.

To demonstrate the feasibility of this fitting routine using clumpy models, we selected 11 representative Ly $\alpha$ spectra from the high SB regions, as shown in Fig. 5. All of the spectra presented have been smoothed spatially by a 3 pixel $\times 3$ pixel boxcar and were extracted by using an $R=3$ pixel $(0.9 \operatorname{arcsec})$ aperture. For each spectrum, we used 1500 live points (the initial randomly drawn samples from the prior) to calculate a set of clumpy models via linear flux interpolation on the grid and convolved with the KCWI LSF before comparing them to the observed Ly $\alpha$ profiles. The best-fitting spectra are also shown in Fig. 5.

\subsection{Results}

In Fig. 5, one can see that most of the model fits match the observations reasonably well. The values of the fitted parameters

The application of a more advanced, self-consistent model is beyond the scope of this work but will be explored in the future.

${ }^{13}$ Note that we did not employ the commonly used 'core-skipping' technique, as it may cause artifacts in a multiphase medium. 
Table 4. Fitted parameters of the multiphase clumpy model and derived quantities.

\begin{tabular}{|c|c|c|c|c|c|c|c|c|c|c|c|c|}
\hline \multirow[b]{2}{*}{$\begin{array}{l}\text { No. } \\
\text { (1) }\end{array}$} & \multirow[b]{2}{*}{$\begin{array}{c}\text { RA (J2000) } \\
\text { (2) }\end{array}$} & \multirow[b]{2}{*}{$\begin{array}{l}\text { Dec. }(\mathrm{J} 2000) \\
\text { (3) }\end{array}$} & \multirow[b]{2}{*}{$\begin{array}{c}\log n_{\mathrm{HI}, \mathrm{ICM}} \\
\left(\mathrm{cm}^{-3}\right) \\
(4)\end{array}$} & \multicolumn{4}{|c|}{ Fitted parameters } & \multirow[b]{2}{*}{$\begin{array}{c}\Delta v \\
\left(\mathrm{~km} \mathrm{~s}^{-1}\right) \\
(9)\end{array}$} & \multicolumn{2}{|c|}{ Derived parameters } & \multicolumn{2}{|c|}{ Moments } \\
\hline & & & & $\begin{array}{l}F_{\mathrm{V}} \\
(5)\end{array}$ & $\begin{array}{c}\sigma_{\mathrm{cl}} \\
\left(\mathrm{km} \mathrm{s}^{-1}\right) \\
(6)\end{array}$ & $\begin{array}{c}v_{\mathrm{cl}} \\
\left(\mathrm{km} \mathrm{s}^{-1}\right) \\
(7)\end{array}$ & $\begin{array}{c}v_{\mathrm{ICM}} \\
\left(\mathrm{km} \mathrm{s}^{-1}\right) \\
(8)\end{array}$ & & $\begin{array}{c}f_{\mathrm{cl}} / f_{\mathrm{cl}, \text { crit }} \\
(10)\end{array}$ & $\begin{array}{c}\log \tau_{0, \mathrm{ICM}} \\
(11)\end{array}$ & $\begin{array}{c}v_{\mathrm{Ly} \alpha} \\
\left(\mathrm{km} \mathrm{s}^{-1}\right) \\
(12)\end{array}$ & $\begin{array}{c}\sigma_{\mathrm{Ly} \alpha} \\
\left(\mathrm{km} \mathrm{s}^{-1}\right) \\
(13)\end{array}$ \\
\hline 1 & $22: 17: 26.214$ & $+00: 12: 54.85$ & $-7.13_{-0.13}^{+0.10}$ & $0.37_{-0.16}^{+0.15}$ & $131_{-94}^{+167}$ & $773_{-159}^{+26}$ & $8_{-7}^{+19}$ & $\begin{array}{l}-195_{-49}^{+34} \\
\end{array}$ & $12.8_{-10.2}^{+17.2}$ & $0.8_{-0.1}^{+0.1}$ & -98.2 & 373.9 \\
\hline 2 & $22: 17: 26.214$ & $+00: 12: 53.05$ & $-6.47_{-0.80}^{+0.40}$ & $0.37_{-0.25}^{+0.22}$ & $242_{-104}^{+112}$ & $497_{-203}^{+217}$ & $444_{-60}^{+131}$ & $-268_{-88}^{+60}$ & $0.8_{-0.7}^{+1.8}$ & $-3.6_{-0.8}^{+1.0}$ & -54.2 & 324.2 \\
\hline 3 & $22: 17: 26.294$ & $+00: 12: 46.75$ & $-7.01_{-0.88}^{+0.88}$ & $0.32_{-0.21}^{+0.26}$ & $111_{-102}^{+263}$ & $392_{-259}^{+385}$ & $348_{-96}^{+156}$ & $43_{-129}^{+92}$ & $0.1_{-0.1}^{+6.9}$ & $-2.1_{-2.8}^{+1.5}$ & 193.4 & 254.1 \\
\hline 4 & $22: 17: 26.054$ & $+00: 12: 41.65$ & $-6.15_{-0.31}^{+0.14}$ & $0.18_{-0.07}^{+0.25}$ & $705_{-392}^{+91}$ & $689_{-494}^{+108}$ & $653_{-62}^{+123}$ & $-200_{-90}^{+48}$ & $0.7_{-0.6}^{+0.7}$ & $-4.2_{-0.3}^{+0.2}$ & 20.6 & 339.2 \\
\hline 5 & $22: 17: 25.954$ & $+00: 12: 40.45$ & $-6.94_{-0.57}^{+0.09}$ & $0.23_{-0.03}^{+0.09}$ & $534_{-91}^{+53}$ & $474_{-463}^{+205}$ & $7_{-7}^{+58}$ & $-69_{-27}^{+139}$ & $2.0_{-0.4}^{+1.4}$ & $1.0_{-0.7}^{+0.1}$ & 81.5 & 435.2 \\
\hline 6 & $22: 17: 25.874$ & $+00: 12: 38.05$ & $-6.90_{-0.26}^{+0.10}$ & $0.18_{-0.02}^{+0.04}$ & $455_{-47}^{+48}$ & $208_{-196}^{+234}$ & $48_{-28}^{+46}$ & $-66_{-32}^{+58}$ & $1.8_{-0.3}^{+0.5}$ & $1.0_{-0.4}^{+0.1}$ & 6.2 & 394.9 \\
\hline 7 & $22: 17: 25.954$ & $+00: 12: 38.05$ & $-7.02_{-0.90}^{+0.14}$ & $0.29_{-0.06}^{+0.19}$ & $437_{-41}^{+48}$ & $376_{-188}^{+127}$ & $16_{-15}^{+391}$ & $-100_{-44}^{+53}$ & $3.0_{-0.8}^{+1.6}$ & $0.9_{-5.1}^{+0.1}$ & 46.3 & 402.5 \\
\hline 8 & $22: 17: 26.034$ & $+00: 12: 37.15$ & $-7.90_{-0.10}^{+0.53}$ & $0.51_{-0.05}^{+0.04}$ & $498_{-39}^{+77}$ & $228_{-76}^{+98}$ & $435_{-112}^{+138}$ & $-162_{-65}^{+61}$ & $4.3_{-0.8}^{+0.5}$ & $-4.8_{-1.0}^{+2.2}$ & 14.6 & 448.8 \\
\hline 9 & $22: 17: 26.034$ & $+00: 12: 35.95$ & $-7.83_{-0.16}^{+0.72}$ & $0.56_{-0.07}^{+0.03}$ & $475_{-31}^{+47}$ & $276_{-123}^{+68}$ & $425_{-108}^{+108}$ & $-181_{-62}^{+89}$ & $4.6_{-0.6}^{+0.5}$ & $-4.6_{-1.1}^{+2.5}$ & 54.3 & 452.9 \\
\hline 10 & $22: 17: 25.694$ & $+00: 12: 34.45$ & $-7.49_{-0.48}^{+0.33}$ & $0.28_{-0.11}^{+0.15}$ & $353_{-32}^{+110}$ & $270_{-246}^{+75}$ & $302_{-70}^{+91}$ & $-135_{-73}^{+109}$ & $2.8_{-1.0}^{+1.1}$ & $-1.9_{-2.0}^{+1.1}$ & -19.6 & 377.1 \\
\hline 11 & $22: 17: 25.634$ & $+00: 12: 34.75$ & $-7.32_{-0.64}^{+0.41}$ & $0.19_{-0.05}^{+0.33}$ & $453_{-111}^{+95}$ & $268_{-256}^{+309}$ & $151_{-95}^{+260}$ & $-9_{-180}^{+69}$ & $1.9_{-0.7}^{+2.6}$ & $0.0_{-4.3}^{+0.9}$ & 44.7 & 378.4 \\
\hline
\end{tabular}

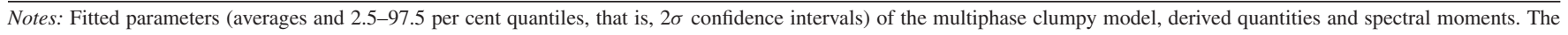

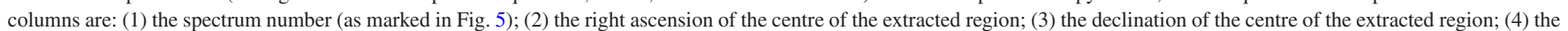

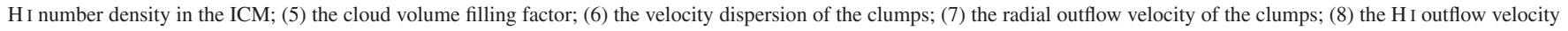

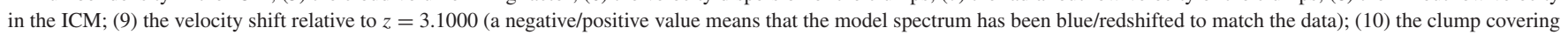

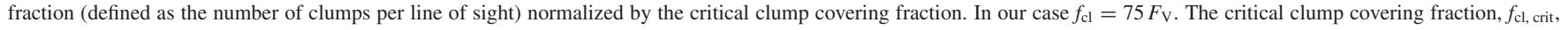

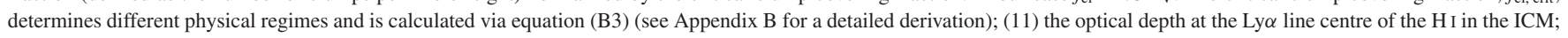
(12) the first moment of the centre of the extracted region; and (13) the second moment of the centre of the extracted region (corrected for KCWI LSF).

are presented in Table 4, and the derived joint and marginal posterior probability distributions are presented in Appendix D. We find that different parameters affect the model spectra in different ways $n_{\mathrm{HI}, \text { ICM }}$ determines the overall shape of the spectrum and the depth of the intensity minimum near the systemic velocity (the 'trough'); $F_{\mathrm{V}}$ and $v_{\mathrm{cl}}$ determine the shapes and strengths of the peak(s); $\sigma_{\mathrm{cl}}$ determines the width of the spectrum; $v_{\text {ICM }}$ determines the location of the main peak and the trough; and $\Delta v$ shifts the spectrum in the velocity dimension. In Fig. 6, we illustrate the impact of each parameter on the emergent model spectrum. Each parameter was varied individually while others were kept fixed to the best-fitting parameter values.

Specifically, spectra 1 and 2 (near the LBG C15) are clearly single-peaked, although spectrum 1 has a subdominant red bump. Interestingly, although spectrum 1 appears to be broader and has a larger $\sigma_{\mathrm{Ly} \alpha}$ on the moment map, it actually requires a smaller $\sigma_{\mathrm{cl}}$ $\left(\sim 150 \mathrm{~km} \mathrm{~s}^{-1}\right)$ than spectrum 2 . The fit of spectrum 1 has a very large $v_{\mathrm{cl}}\left(\sim 800 \mathrm{~km} \mathrm{~s}^{-1}\right)$ and a negligible $v_{\mathrm{ICM}}$, whereas the fit of spectrum 2 has comparable $v_{\mathrm{cl}}$ and $v_{\mathrm{ICM}}$ (both $\sim 500 \mathrm{~km} \mathrm{~s}^{-1}$ ). This is due to the fact that multiphase outflows increase the asymmetry as well as the width of the spectra, and this degeneracy is not captured by the moment analysis. In particular, for spectrum 1 , the optical depth at the Ly $\alpha$ line centre $\tau_{0, \mathrm{ICM}}{ }^{14} \sim 1$, so the Ly $\alpha$ photons likely interact with the ICM prior to the clumps (as opposed to spectrum 2 where $\tau_{0, \text { ICM }} \ll 1$ ). This implies that photons can then scatter approximately orthogonally off the clumps (see Appendix B), which yields an additional broadening of the spectrum that is larger than $\sigma_{\mathrm{cl}}$. The derived $\Delta v\left(\sim-200 \mathrm{~km} \mathrm{~s}^{-1}\right)$ is also consistent with the $\mathrm{C} 15$ systemic redshift $(z=3.0975)$ measured from [O III]. Also note that although spectrum 1 exhibits a blue dominant peak and a smaller red 'hump' (which is commonly interpreted as a signature of inflows), our outflow model has successfully reproduced the observed line profile.

\footnotetext{
${ }^{14} \mathrm{Here}, \tau_{0, \mathrm{ICM}}=n_{\mathrm{HI}, \mathrm{ICM}} r_{\mathrm{gal}} \sigma_{\mathrm{HI}}\left(T_{\mathrm{ICM}}, v_{\mathrm{ICM}}\right)$, where $\sigma_{\mathrm{HI}}\left(T_{\mathrm{ICM}}, v_{\mathrm{ICM}}\right)$ is the
} Ly $\alpha$ absorption cross-section at $\mathrm{HI}$ temperature $T_{\mathrm{ICM}}$ and velocity $v_{\mathrm{ICM}}$.
Spectrum 3 has a fairly narrow main peak, which yields the lowest $\sigma_{\mathrm{cl}}\left(\sim 100 \mathrm{~km} \mathrm{~s}^{-1}\right)$ of all 11 sampled spectra. The fit has comparable $v_{\mathrm{cl}}$ and $v_{\mathrm{ICM}}\left(\right.$ both $\left.\sim 350 \mathrm{~km} \mathrm{~s}^{-1}\right)$ and a small $\Delta v\left(\sim 50 \mathrm{~km} \mathrm{~s}^{-1}\right)$. It also has some dubious emission on the far blue side, which is not captured by the clumpy models. Increasing $\sigma_{\mathrm{cl}}$ in order to include both the red and blue peaks could potentially provide a better fit but would lack physical motivation.

Spectra 4 and 5 (the tail of the tadpole) both possess two comparable peaks and a trough in the middle. The best fit of the former is single-peaked, while the latter is double-peaked and captured the trough. Both fits have very large $\sigma_{\mathrm{cl}}\left(>500 \mathrm{~km} \mathrm{~s}^{-1}\right)$ to account for the line widths. The fit of spectrum 4 has comparably large $v_{\mathrm{cl}}$ and $v_{\mathrm{ICM}}$ (both $\sim 700 \mathrm{~km} \mathrm{~s}^{-1}$ ), whereas spectrum 5 has a $v_{\mathrm{cl}}$ of $\sim 500 \mathrm{~km} \mathrm{~s}^{-1}$ and a negligible $v_{\text {ICM }}$.

Spectra 6 and 7 (the body of the tadpole) are both multipeaked and dominated by a red peak. The best fits both have $\sigma_{\mathrm{cl}} \sim 450 \mathrm{~km} \mathrm{~s}^{-1}$. They have moderate $v_{\mathrm{cl}}\left(\sim 200\right.$ and $400 \mathrm{~km} \mathrm{~s}^{-1}$, respectively) and small $v_{\text {ICM }}<50 \mathrm{~km} \mathrm{~s}^{-1}$ (dictated by the location of the absorption features).

Spectra 8 and 9 (the head of the tadpole, near ALMA-a) are both very broad $\left(\sigma_{\mathrm{cl}} \sim 500 \mathrm{~km} \mathrm{~s}^{-1}\right)$ and red-dominant double-peaked with a deep trough between two peaks. They both have high $F_{\mathrm{V}}(\sim 0.5)$ and moderate $v_{\mathrm{cl}}\left(\sim 250 \mathrm{~km} \mathrm{~s}^{-1}\right)$ and $v_{\mathrm{ICM}}\left(\sim 400 \mathrm{~km} \mathrm{~s}^{-1}\right)$.

Spectra 10 and 11 (near the LBG C11) are also red-dominant double-peaked, although with slightly narrower line widths $\left(\sigma_{\mathrm{cl}}\right.$ $\left.\sim 400 \mathrm{~km} \mathrm{~s}^{-1}\right)$. Compared with spectra 8 and 9, they have lower $F_{\mathrm{V}}(<0.3)$, comparable $v_{\mathrm{cl}}\left(\sim 250 \mathrm{~km} \mathrm{~s}^{-1}\right)$ and lower $v_{\mathrm{ICM}}$ $\left(<300 \mathrm{~km} \mathrm{~s}^{-1}\right)$. The derived $\Delta v$ of spectrum $10\left(\sim-150 \mathrm{~km} \mathrm{~s}^{-1}\right)$ is also consistent with the $\mathrm{C} 11$ systemic redshift $(z=3.0980 \pm$ 0.0001) measured from [O III]. Notably, the prominent double peak profiles in this region require a considerable outflow velocity for both the clumps and the ICM. This strongly suggests the presence of outflows, which is consistent with the indication of the large global velocity offsets between Ly $\alpha$, [O III], and Si II.

It is noteworthy that although the observed $v_{\mathrm{Ly} \alpha}$ is fairly small in many positions (e.g. spectra 4-9), large $\sigma_{\mathrm{cl}}, v_{\mathrm{cl}}$ and and non-zero 


\section{Best-fit Parameters: $n_{\mathrm{ICM}}=3 \times 10^{-8} \mathrm{~cm}^{-3}, F_{\mathrm{v}}=0.28, \sigma_{\mathrm{cl}}=356 \mathrm{~km} / \mathrm{s}$ $v_{\mathrm{Cl}}=269 \mathrm{~km} / \mathrm{s}, v_{\mathrm{ICM}}=299 \mathrm{~km} / \mathrm{s}, \triangle v=-132 \mathrm{~km} / \mathrm{s}$}
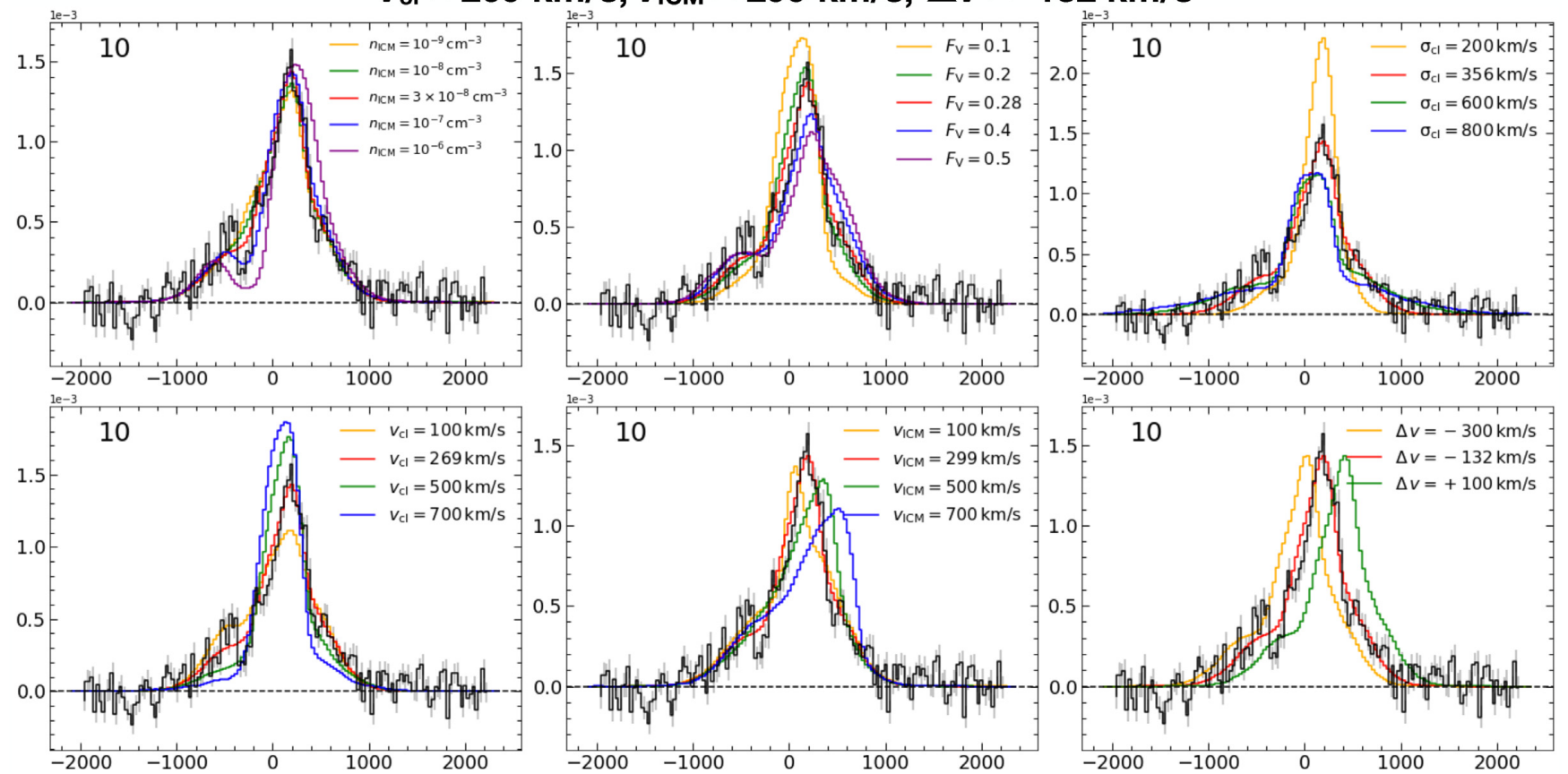

Figure 6. The effects of each individual physical parameter in $\left[n_{\mathrm{HI}}\right.$ ICM $\left., F_{\mathrm{V}}, \sigma_{\mathrm{cl}}, v_{\mathrm{cl}}, v_{\mathrm{ICM}}, \Delta v\right]$ (taking spectrum 10 as an example). From the top left to the bottom right panel, one parameter is varied at a time (as shown in lines and labelled with different colours) and others are fixed (to the best-fitting parameter values of spectrum 10, see Table 4). The red line in each panel represents the best-fitting model of spectrum 10 . The $x$-axis is the velocity (in $\mathrm{km} \mathrm{s}^{-1}$ ) with respect to the local systemic redshift of spectrum 10, and the $y$-axis is the normalized line flux. It can be seen that different parameters affect the model spectra

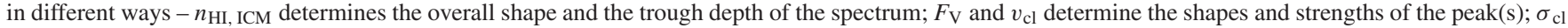
determines the width of the spectrum; $v_{\text {ICM }}$ determines the location of the main peak and the trough; and $\Delta v$ shifts the spectrum in the velocity dimension.

$v_{\text {ICM }}$ are still preferred by the broad, asymmetric Ly $\alpha$ profiles. ${ }^{15}$ This concerns us that the first moment does not fully capture the gas kinematic information encoded in the Ly $\alpha$ profiles. Second moments may be helpful in quantifying the line widths, whose possible physical interpretation is the random velocity dispersion of H I clumps. Furthermore, the outflow velocity (parametrized as $v_{\mathrm{cl}}$ and $v_{\text {ICM }}$ in the model) may be difficult to determine directly from the observed spectra (especially for complex Ly $\alpha$ profiles), but might be retrieved using realistic radiative transfer modelling.

In addition, we note that both the average and the standard deviation of all the derived $\Delta v$ are fairly small $\left(\langle\Delta v\rangle=-122 \mathrm{~km} \mathrm{~s}^{-1}\right.$, $\left.\sigma(\Delta v)=87 \mathrm{~km} \mathrm{~s}^{-1}\right)$, despite the large outflow velocities indicated by many of the Ly $\alpha$ profiles. This corresponds to an average systemic redshift of LAB1, $\left\langle z_{\text {sys }}\right\rangle=3.0983 \pm 0.0004$.

We caution that the effect of $\operatorname{Ly} \alpha$ absorption from the intergalactic medium (IGM) is not modelled in this work. It is expected that at $z \sim 3$ this effect is in general non-negligible on the blue side of the spectrum (Dijkstra, Lidz \& Wyithe 2007; Laursen, Sommer-Larsen \& Razoumov 2011). However, we do not expect the effect of the IGM to be significant here, as it would cause sharp absorption troughs and yield multiple peaks, which should be clearly visible given the widths of the observed spectra (see Byrohl \& Gronke 2020 for a discussion of this effect) instead of simply attenuating the spectrum smoothly.

${ }^{15}$ This is also notable in cases where an [O III] measurement is available and the velocity offset between Ly $\alpha$ and [O III] is close to zero (e.g. spectra 1 and 2).
To recap, the main results of our analysis are:

(i) The observed Ly $\alpha$ spectra require relatively few clumps per line of sight $\left(f_{\mathrm{cl}} \lesssim f_{\mathrm{cl} \text {, crit }}\right)$ as they have significant fluxes at the line centre. Therefore, they are very different from the spectra of most Ly $\alpha$ emitting galaxies at essentially all redshifts (e.g. Steidel et al. 2010; Erb et al. 2014; Trainor et al. 2015; Yang et al. 2016, 2017; Gronke 2017; Orlitová et al. 2018), which can usually be reproduced by a uniform medium (e.g. the 'shell model') ${ }^{16}$ or by a multiphase medium with a large number H I clumps $\left(f_{\mathrm{cl}} \gg f_{\mathrm{cl} \text {, crit }}\right)$.

(ii) The velocity dispersion of the scattering clumps yields a broadening of the spectra from the intrinsic line width $\sigma_{\mathrm{i}} \sim 13 \mathrm{~km} \mathrm{~s}^{-1}$ to $\gg 100 \mathrm{~km} \mathrm{~s}^{-1}$ as observed. This is possible when $f_{\mathrm{cl}} \sim \alpha f_{\mathrm{cl}}$, crit with $\alpha \sim$ a few. ${ }^{17}$ Such a process may be crucial in galaxies where the observed Ly $\alpha$ line is always broader, usually by at least a factor of 2 , than the corresponding non-resonant lines such as [O III], $\mathrm{H} \alpha$ or $\mathrm{H} \beta$ (e.g. Orlitová et al. 2018).

(iii) While the widths of the spectra are set primarily by the velocity dispersion of the clumps, that is, $\sigma_{\mathrm{Ly} \alpha} \sim \sigma_{\mathrm{cl}}$, we found that the clump bulk outflow can also cause additional broadening, as seen in spectrum 1. In this case, one might naively assume that the photons

\footnotetext{
${ }^{16}$ The success of shell model fitting may no longer be achieved when the model parameters are further constrained by additional observations (e.g. optical emission lines or UV absorption lines, see Orlitová et al. 2018).

${ }^{17}$ Note that the scattering off the surface of the clumps broadens the spectrum as long as $\sigma_{\mathrm{cl}}>\sigma_{\mathrm{i}}$ (and $f_{\mathrm{cl}} \sim f_{\mathrm{cl} \text {, crit }}$ ). Hence, the emergent spectra are insensitive to the exact value of $\sigma_{\mathrm{i}}$.
} 
do not interact with the clumps due to their large velocity offsets $\left(v_{\mathrm{cl}}\right.$ $\gg \sigma_{\mathrm{cl}}$ ). However, if $\tau_{0 \text {, ICM }} \gtrsim 1$, the photons may first interact with the ICM, which significantly reduces the parallel component of $v_{\mathrm{cl}}\left(v_{\mathrm{cl}, \ldots}\right)$ appearing to the photons, and hence greatly increases the optical depth. This result suggests that we may have interpreted our model too naively (e.g. using single-scattering approximation), especially considering that the kinematics of our model are clearly simplistic and not strictly hydrodynamically stable (we usually expect $v_{\mathrm{cl}} \sim$ $v_{\text {ICM }}$, i.e. the clouds are entrained by the local flow, see e.g. Klein, McKee \& Colella 1994; Li et al. 2020). ${ }^{18}$ Moreover, although we found that significant outflow velocities $\left(\gtrsim 100 \mathrm{~km} \mathrm{~s}^{-1}\right)$ are required to reproduce the observed spectra, the exact values may still be subject to considerable uncertainties, due to the internal degeneracies and the presumably more complicated kinematics in reality (e.g. Steidel et al. 2010 show that gas outflows even within the same galaxy have a range of velocities that goes from 0 to $800 \mathrm{~km} \mathrm{~s}^{-1}$ with varying effective optical depths).

(iv) In our best-fitting spectra, the H I in the ICM is responsible for the absorption feature close to the line centre (cf. spectrum 5 or 6). However, several tentative absorption features can be present in a single spectrum (e.g. spectra 5, 6, 7, and 9), and they are not captured simultaneously by our model. These multiple features might be caused by the $\mathrm{H}$ I in the outer CGM/IGM, where the probability of backscattering into the line of sight is negligible.

The derived values of our fitted parameters also fit into a broader picture in at least two ways:

(i) The $\sigma_{\mathrm{cl}}$ values correspond to reasonable dark matter halo masses. The dynamical mass of the LAB1 halo can be estimated from the velocity dispersion and physical size (assuming spherical symmetry):

$M_{\mathrm{dyn}}=\frac{3 \sigma_{\mathrm{cl}}^{2} R}{G}=6.9 \times 10^{9}\left(\frac{\sigma_{\mathrm{cl}}}{100 \mathrm{~km} \mathrm{~s}^{-1}}\right)^{2}\left(\frac{R}{\mathrm{kpc}}\right) \mathrm{M}_{\odot}$.

Taking $R \sim 100 \mathrm{kpc}$, the highest $\sigma_{\mathrm{cl}}\left(\sim 700 \mathrm{~km} \mathrm{~s}^{-1}\right)$ corresponds to $M_{\text {dyn }} \sim 10^{13.5} \mathrm{M}_{\odot}$. This result is consistent with the predicted halo masses from the Millennium simulations at $z=3.06$. As calculated by Kubo et al. (2016), the halo masses range from $10^{12.2}$ to $10^{14.0} \mathrm{M}_{\odot}$ (with median $10^{13.2} \mathrm{M}_{\odot}$ ) for the De Lucia \& Blaizot (2007) model, and from $10^{12.4}$ to $10^{14.1} \mathrm{M}_{\odot}$ (with median $10^{13.2} \mathrm{M}_{\odot}$ ) for the Guo et al. (2011) model.

(ii) The $v_{\mathrm{cl}}$ values correspond to reasonable survival times of the clumps. Here, we consider two different criteria proposed by Li et al. (2020) and Gronke \& Oh (2018), respectively. Following Li et al. (2020), assuming the ionized medium has H II number density $n_{\text {hot }}$ $=0.01 \mathrm{~cm}^{-3}$, an outflow velocity $v_{\mathrm{cl}} \sim 500 \mathrm{~km} \mathrm{~s}^{-1}$ corresponds to a cloud lifetime $t_{\text {life }} \sim 100 \mathrm{Myr}$. The cooling time of the hot medium, $t_{\text {cool, } \mathrm{h}} \sim 30 \mathrm{Myr} \leq t_{\text {life }}$. Whereas following Gronke \& $\mathrm{Oh}$ (2018), the cooling time of the mixing layer $t_{\text {cool, mix }} \sim 3 \mathrm{Myr}$, and the cloud-crushing time $t_{\mathrm{cc}} \sim 1 \mathrm{Myr} \simeq t_{\text {cool, mix }}$, which implies possible survival of the cold gas. So either criterion indicates that the clumps can survive for a fairly long time, and may even grow in mass as they accrete the cooling hot material from the ambient medium.

Recently, Herenz et al. (2020) have reported a significant detection of He II $\lambda 1640$ emission in three regions of LAB1 (which are close to $\mathrm{C} 15$, the tail of the tadpole structure, and $\mathrm{C} 11$, respectively) as well as a non-detection of C IV $\lambda \lambda 1548,1550$ doublet. They have carried out a detailed analysis and concluded that their observed He II/Ly $\alpha$

\footnotetext{
${ }^{18}$ We do see $v_{\text {cl }} \sim v_{\text {ICM }}$ in our fits of spectra $3,4,5,10$, and 11 .
}

and $\mathrm{C}$ IV/Ly $\alpha$ ratios are consistent with cooling radiation, feedbackdriven shocks, and/or photo-ionization from an embedded AGN. We examined our MOSFIRE spectra around these regions but did not find additional rest-UV collisionally excited emission lines near Ly $\alpha$ that are significant, although we do see significant outflow velocities in these three regions (cf. spectra 1, 2, 5, 10, and 11). More observations are needed to distinguish these different powering mechanisms.

In summary, the multiphase clumpy model is versatile enough to reproduce the diverse Ly $\alpha$ morphologies observed. The fitting results are still, not surprisingly, model dependent-different assumptions on the geometry and moving pattern of the H I gas may yield different results. Furthermore, our modelling with parameters of scattering medium varying independently at different locations can be handled in a more self-consistent manner, as in reality the Ly $\alpha$ photons are likely to be scattered by a common, spatially varying medium. None the less, our analysis is a first attempt to model the spatially resolved Ly $\alpha$ profiles in LAB1 with more physically realistic clumpy models. It provides us with insights on the gas kinematics and will serve as the foundation of more advanced radiative transfer modelling in the future. One promising future direction is to use more elaborate clump velocity profiles (e.g. consistent with absorption line observations) which can alter $f_{\mathrm{cl}}$, crit (cf. Appendix B). We will explore such new physical regimes in our future work.

\section{CONCLUSIONS}

We have carried out deep spectroscopic observations of SSA22LAB 1 at $z=3.1$ using KCWI and MOSFIRE. The main conclusions of our analysis are:

(i) By applying matched filtering to the KCWI datacube, we have created a narrow-band Ly $\alpha$ image of LAB1. The most prominent feature is a tadpole-shaped structure, whose 'head' overlaps with one of the ALMA sources yet whose 'tail' does not associate with any identified sources.

(ii) By comparing the spatial distributions and intensities of Ly $\alpha$ and $\mathrm{H} \beta$, we find that recombination of photo-ionized $\mathrm{H}$ I gas followed by resonant scattering is sufficient to explain all the observed $\mathrm{Ly} \alpha / \mathrm{H} \beta$ ratios.

(iii) Using both moment map analysis and MCRT modelling, we have managed to extract physical information from the spatially resolved Ly $\alpha$ profiles. We find that moment maps can be used as a crude indicator of the H I gas kinematics, but realistic MCRT modelling needs to be invoked to extract detailed kinematic information and make physical interpretations. By fitting a set of multiphase, 'clumpy' models to the observed Ly $\alpha$ profiles, we are able to reasonably constrain many physical parameters, namely the H I number density in the ICM, the cloud volume filling factor, the random velocity and outflow velocity of the clumps, the H I outflow velocity of the ICM and the local systemic redshift. Our model has successfully reproduced the diverse Ly $\alpha$ morphologies at different locations, and the main results are: (1) the observed Ly $\alpha$ spectra require relatively few clumps per line of sight $\left(f_{\mathrm{cl}} \lesssim f_{\mathrm{cl} \text {, crit }}\right)$ as they have significant fluxes at the line centre; (2) the velocity dispersion of the scattering clumps yields a significant broadening of the spectra as observed; (3) the clump bulk outflow can also cause additional broadening if $\tau_{0 \text {, ICM }} \gtrsim 1$. In that case, the photons may first interact with the ICM, which significantly reduces the parallel component of clump outflow velocity appearing to the photons, and hence greatly increases the optical depth of the clumps; and (4) the H I in the ICM is responsible for the absorption feature close to the $\operatorname{Ly} \alpha$ line centre. 
We caution that there are still a number of caveats to this study. For example, our MCRT modelling is inherently model dependent, in particular on the specific assumptions about the kinematics of the cold clumps. A combination of results from hydrodynamical simulations and additional observations (e.g. absorption line studies) may help constrain the actual gas kinematics better. We intend to explore these possibilities in our future work.

\section{ACKNOWLEDGEMENTS}

We thank the referee for insightful comments that have significantly improved the paper. We also thank Phil Hopkins for providing us with adequate computational resources. ZL acknowledges Michael Zhang, for his professional guidance and kind company during the excruciating debugging process. The data presented herein were obtained at the W. M. Keck Observatory, which is operated as a scientific partnership among the California Institute of Technology, the University of California, and the National Aeronautics and Space Administration. The Observatory was made possible by the generous financial support of the W. M. Keck Foundation. We are also grateful to the dedicated staff of the W.M. Keck Observatory who keep the instruments and telescopes running effectively. MG was supported by NASA through the NASA Hubble Fellowship grant HST-HF2-51409 awarded by the Space Telescope Science Institute, which is operated by the Association of Universities for Research in Astronomy, Inc., for NASA, under contract NAS526555. Numerical calculations were run on the Caltech compute cluster 'Wheeler,' allocations from XSEDE TG-AST130039 and PRAC NSF.1713353 supported by the NSF, and NASA HEC SMD16-7592. This research made use of Montage. It is funded by the National Science Foundation under grant number ACI-1440620, and was previously funded by the National Aeronautics and Space Administration's Earth Science Technology Office, Computation Technologies Project, under cooperative agreement number NCC5626 between NASA and the California Institute of Technology.

\section{DATA AVAILABILITY}

The data underlying this article will be shared on reasonable request to the corresponding author.

\section{REFERENCES}

Beck M., Scarlata C., Hayes M., Dijkstra M., Jones T. J., 2016, ApJ, 818, 138 Bower R. G. et al., 2004, MNRAS, 351, 63

Byrohl C., Gronke M., 2020, A\&A, 642, L16

Cai Z. et al., 2017, ApJ, 837, 71

Cantalupo S., Porciani C., Lilly S. J., Miniati F., 2005, ApJ, 628, 61

Cantalupo S., Arrigoni-Battaia F., Prochaska J. X., Hennawi J. F., Madau P., 2014, Nature, 506, 63

Chapman S. C., Lewis G. F., Scott D., Richards E., Borys C., Steidel C. C., Adelberger K. L., Shapley A. E., 2001, ApJ, 548, L17

Colbert J. W., Teplitz H., Francis P., Palunas P., Williger G. M., Woodgate B., 2006, ApJ, 637, L89

Davies R., Agudo Berbel A., Wiezorrek E., Ott T., Förster-Schreiber N. M., 2010, Proceedings of the SPIE, Vol. 7735. Society of Photo-optical Instrumentation Engineers, Bellingham, WA, p. $77356 \mathrm{~V}$

De Lucia G., Blaizot J., 2007, MNRAS, 375, 2

Dere K., 2013, Astrophysics Source Code Library, record ascl:1308.017

Dere K. P., Landi E., Mason H. E., Monsignori Fossi B. C., Young P. R., 1997, A\&AS, 125, 149

Dere K. P., Del Zanna G., Young P. R., Landi E., Sutherland R. S., 2019, ApJS, 241, 22
Dessauges-Zavadsky M., D’Odorico S., Schaerer D., Modigliani A., Tapken C., Vernet J., 2010, A\&A, 510, A26

Dey A. et al., 2005, ApJ, 629, 654

Dijkstra M., Kramer R., 2012, MNRAS, 424, 1672

Dijkstra M., Loeb A., 2009, MNRAS, 400, 1109

Dijkstra M., Haiman Z., Spaans M., 2006a, ApJ, 649, 14

Dijkstra M., Haiman Z., Spaans M., 2006b, ApJ, 649, 37

Dijkstra M., Lidz A., Wyithe J. S. B., 2007, MNRAS, 377, 1175

Eide M. B., Gronke M., Dijkstra M., Hayes M., 2018, ApJ, 856, 156

Erb D. K., Bogosavljević M., Steidel C. C., 2011, ApJ, 740, L31

Erb D. K. et al., 2014, ApJ, 795, 33

Fardal M. A., Katz N., Gardner J. P., Hernquist L., Weinberg D. H., Davé R., 2001, ApJ, 562, 605

Faucher-Giguère C.-A., Kereš D., Dijkstra M., Hernquist L., Zaldarriaga M., 2010, ApJ, 725, 633

Forero-Romero J. E., Gronke M., Remolina-Gutiérrez M. C., GaravitoCamargo N., Dijkstra M., 2018, MNRAS, 474, 12

Francis P. J. et al., 1996, ApJ, 457, 490

Furlanetto S. R., Schaye J., Springel V., Hernquist L., 2005, ApJ, 622, 7

Fynbo J. U., Møller P., Warren S. J., 1999, MNRAS, 305, 849

Geach J. E. et al., 2005, MNRAS, 363, 1398

Geach J. E., Smail I., Chapman S. C., Alexand er D. M., Blain A. W., Stott J. P., Ivison R. J., 2007, ApJ, 655, L9

Geach J. E. et al., 2009, ApJ, 700, 1

Geach J. E. et al., 2014, ApJ, 793, 22

Geach J. E. et al., 2016, ApJ, 832, 37

Ginsburg A., Mirocha J., 2011, Astrophysics Source Code Library, record ascl:1109.001

Goerdt T., Dekel A., Sternberg A., Ceverino D., Teyssier R., Primack J. R., 2010, MNRAS, 407, 613

Gronke M., 2017, A\&A, 608, A139

Gronke M., Oh S. P., 2018, MNRAS, 480, L111

Gronke M., Dijkstra M., 2016, ApJ, 826, 14

Gronke M., Dijkstra M., McCourt M., Peng Oh S., 2017, A\&A, 607, A71

Guo Q. et al., 2011, MNRAS, 413, 101

Haiman Z., Rees M. J., 2001, ApJ, 556, 87

Haiman Z., Spaans M., Quataert E., 2000, ApJ, 537, L5

Hansen M., Oh S. P., 2006, MNRAS, 367, 979

Hashimoto T. et al., 2015, ApJ, 812, 157

Hayes M., Scarlata C., Siana B., 2011, Nature, 476, 304

Heckman T. M., Armus L., Miley G. K., 1990, ApJS, 74, 833

Hennawi J. F., Prochaska J. X., Kollmeier J., Zheng Z., 2009, ApJ, 693, L49

Hennawi J. F., Prochaska J. X., Cantalupo S., Arrigoni-Battaia F., 2015, Science, 348, 779

Herenz E. C., Wisotzki L., 2017, A\&A, 602, A111

Herenz E. C., Hayes M., Scarlata C., 2020, A\&A, 642, A55

Hine N. K., Geach J. E., Alexander D. M., Lehmer B. D., Chapman S. C., Matsuda Y., 2016, MNRAS, 455, 2363

Hummels C. B. et al., 2019, ApJ, 882, 156

Keel W. C., Cohen S. H., Windhorst R. A., Waddington I., 1999, AJ, 118, 2547

Klein R. I., McKee C. F., Colella P., 1994, ApJ, 420, 213

Kubo M., Yamada T., Ichikawa T., Kajisawa M., Matsuda Y., Tanaka I., 2015, ApJ, 799, 38

Kubo M., Yamada T., Ichikawa T., Kajisawa M., Matsuda Y., Tanaka I., Umehata H., 2016, MNRAS, 455, 3333

Kulas K. R., Shapley A. E., Kollmeier J. A., Zheng Z., Steidel C. C., Hainline K. N., 2012, ApJ, 745, 33

Laursen P., Sommer-Larsen J., Razoumov A. O., 2011, ApJ, 728, 52

Laursen P., Duval F., Östlin G., 2013, ApJ, 766, 124

Li Z., Hopkins P. F., Squire J., Hummels C., 2020, MNRAS, 492, 1841

Luridiana V., Morisset C., Shaw R. A., 2015, A\&A, 573, A42

Martin C., Moore A., Morrissey P., Matuszewski M., Rahman S., Adkins S., Epps H., 2010, in McLean I. S., Ramsay S. K., Takami H., eds, Society of Photo-Optical Instrumentation Engineers (SPIE) Conference Series. Society of Photo-optical Instrumentation Engineers, Bellingham, WA, p. 77350M

Matsuda Y. et al., 2004, AJ, 128, 569 
Matsuda Y., Iono D., Ohta K., Yamada T., Kawabe R., Hayashino T., Peck A. B., Petitpas G. R., 2007, ApJ, 667, 667

Matsuda Y. et al., 2011, MNRAS, 410, L13

McLean I. S. et al., 2010, in McLean I. S., Ramsay S. K., Takami H., eds, Society of Photo-Optical Instrumentation Engineers (SPIE) Conference Series. Society of Photo-optical Instrumentation Engineers, Bellingham, WA, p. 77351E

McLean I. S. et al., 2012, in McLean I. S., Ramsay S. K., Takami H., eds, Society of Photo-Optical Instrumentation Engineers (SPIE) Conference Series. Society of Photo-optical Instrumentation Engineers, Bellingham, WA, p. 84460J

McLinden E. M. et al., 2011, ApJ, 730, 136

McLinden E. M., Malhotra S., Rhoads J. E., Hibon P., Weijmans A.-M., Tilvi V., 2013, ApJ, 767, 48

Mori M., Umemura M., Ferrara A., 2004, ApJ, 613, L97

Morrissey P., et al., 2012, in McLean I. S., Ramsay S. K., Takami H., eds, Society of Photo-Optical Instrumentation Engineers (SPIE) Conference Series. Society of Photo-optical Instrumentation Engineers, Bellingham, WA, p. 844613

Nestor D. B., Shapley A. E., Steidel C. C., Siana B., 2011, ApJ, 736, 18

Neufeld D. A., 1991, ApJ, 370, L85

Nilsson K. K., Fynbo J. P. U., Møller P., Sommer-Larsen J., Ledoux C., 2006, A\&A, 452, L23

Ohyama Y. et al., 2003, ApJ, 591, L9

Orlitová I., Verhamme A., Henry A., Scarlata C., Jaskot A., Oey M. S., Schaerer D., 2018, A\&A, 616, A60

Ott T., 2012, Astrophysics Source Code Library, record ascl:1210.019

Ouchi M. et al., 2009, ApJ, 696, 1164

Planck Collaboration et al., 2020, A\&A, 641, A6

Prescott M. K. M., Kashikawa N., Dey A., Matsuda Y., 2008, ApJ, 678, L77

Prescott M. K. M., Dey A., Jannuzi B. T., 2009, ApJ, 702, 554

Prescott M. K. M., Dey A., Jannuzi B. T., 2012, ApJ, 748, 125

Rivera-Thorsen T. E. et al., 2017, A\&A, 608, L4

Rosdahl J., Blaizot J., 2012, MNRAS, 423, 344

Saito T., Shimasaku K., Okamura S., Ouchi M., Akiyama M., Yoshida M., 2006, ApJ, 648, 54

Saito T., Shimasaku K., Okamura S., Ouchi M., Akiyama M., Yoshida M., Ueda Y., 2008, ApJ, 675, 1076

Scarlata C. et al., 2009, ApJ, 706, 1241

Schaerer D., Verhamme A., 2008, A\&A, 480, 369

Skilling J., 2004, in Fischer R., Preuss R., Toussaint U. V., eds, AIP Conference Proceedings, Vol. 735. American Institute of Physics, Melville, NY, p. 395

Skilling J., 2006, Bayesian Anal., 1, 833

Smith D. J. B., Jarvis M. J., 2007, MNRAS, 378, L49

Smith D. J. B., Jarvis M. J., Lacy M., Martínez-Sansigre A., 2008, MNRAS, 389,799

Speagle J. S., 2020, MNRAS, 493, 3132

Steidel C. C., Adelberger K. L., Dickinson M., Giavalisco M., Pettini M., Kellogg M., 1998, ApJ, 492, 428

Steidel C. C., Adelberger K. L., Shapley A. E., Pettini M., Dickinson M., Giavalisco M., 2000, ApJ, 532, 170

Steidel C. C., Adelberger K. L., Shapley A. E., Pettini M., Dickinson M., Giavalisco M., 2003, ApJ, 592, 728

Steidel C. C., Erb D. K., Shapley A. E., Pettini M., Reddy N., Bogosavljević M., Rudie G. C., Rakic O., 2010, ApJ, 717, 289

Steidel C. C., Bogosavljević M., Shapley A. E., Kollmeier J. A., Reddy N. A., Erb D. K., Pettini M., 2011, ApJ, 736, 160
Steidel C. C. et al., 2014, ApJ, 795, 165

Strom A. L., Steidel C. C., Rudie G. C., Trainor R. F., Pettini M., Reddy N. A., 2017, ApJ, 836, 164

Taniguchi Y., Shioya Y., 2000, ApJ, 532, L13

Taniguchi Y., Shioya Y., Kakazu Y., 2001, ApJ, 562, L15

Trainor R. F., Steidel C. C., Strom A. L., Rudie G. C., 2015, ApJ, 809, 89

Trebitsch M., Verhamme A., Blaizot J., Rosdahl J., 2016, A\&A, 593, A122

Uchimoto Y. K. et al., 2008, PASJ, 60, 683

Uchimoto Y. K. et al., 2012, ApJ, 750, 116

Umehata H. et al., 2017, ApJ, 834, L16

Vanzella E. et al., 2010, A\&A, 513, A20

Verhamme A., Schaerer D., Maselli A., 2006, A\&A, 460, 397

Verhamme A., Schaerer D., Atek H., Tapken C., 2008, A\&A, 491, 89

Webb T. M. A., Yamada T., Huang J. S., Ashby M. L. N., Matsuda Y., Egami E., Gonzalez M., Hayashimo T., 2009, ApJ, 692, 1561

Weijmans A.-M., Bower R. G., Geach J. E., Swinbank A. M., Wilman R. J., de Zeeuw P. T., Morris S. L., 2010, MNRAS, 402, 2245

Yang Y., Zabludoff A., Tremonti C., Eisenstein D., Davé R., 2009, ApJ, 693 , 1579

Yang Y., Zabludoff A., Eisenstein D., Davé R., 2010, ApJ, 719, 1654

Yang H., Malhotra S., Gronke M., Rhoads J. E., Dijkstra M., Jaskot A., Zheng Z., Wang J., 2016, ApJ, 820, 130

Yang H. et al., 2017, ApJ, 844, 171

Zheng Z., Miralda-Escudé J., 2002, ApJ, 578, 33

\section{SUPPORTING INFORMATION}

Supplementary data are available at MNRAS online.

\section{Appendix_D_merged.pdf}

Please note: Oxford University Press is not responsible for the content or functionality of any supporting materials supplied by the authors. Any queries (other than missing material) should be directed to the corresponding author for the article.

\section{APPENDIX A: GAUSSIAN FITS TO THE [O III] PROFILES OF LBGS C11 AND C15}

In order to determine the systemic redshifts of two LBGs, C11 and C15, we fitted the [O III] profiles (5008.24 $\AA$ ) with single Gaussians. The [O III] emission is spatially integrated over the ranges indicated by black solid arrows in Fig. 3, which include all the significant [O III] emission of $\mathrm{C} 11$ and $\mathrm{C} 15$.

We used the PYSPECKIT package (Ginsburg \& Mirocha 2011) to fit the [O III] profiles with a single Gaussian model:

$F_{\lambda}=F_{0} e^{-\frac{\left(\lambda-\lambda_{0}\right)^{2}}{2 \sigma^{2}}}$

The fitting results and derived values with $1 \sigma$ uncertainties of the free parameters $\left(F_{0}, \lambda_{0}\right.$, and $\left.\sigma\right)$ are shown in Fig. A1. The systemic redshifts of $\mathrm{C} 11$ and $\mathrm{C} 15$ are therefore determined to be $z(\mathrm{C} 11)$ $=3.0980 \pm 0.0001$ and $z(\mathrm{C} 15)=3.0975 \pm 0.0001$ (accounting for the typical redshift precision achieved by MOSFIRE measurements reported in Steidel et al. 2014). 

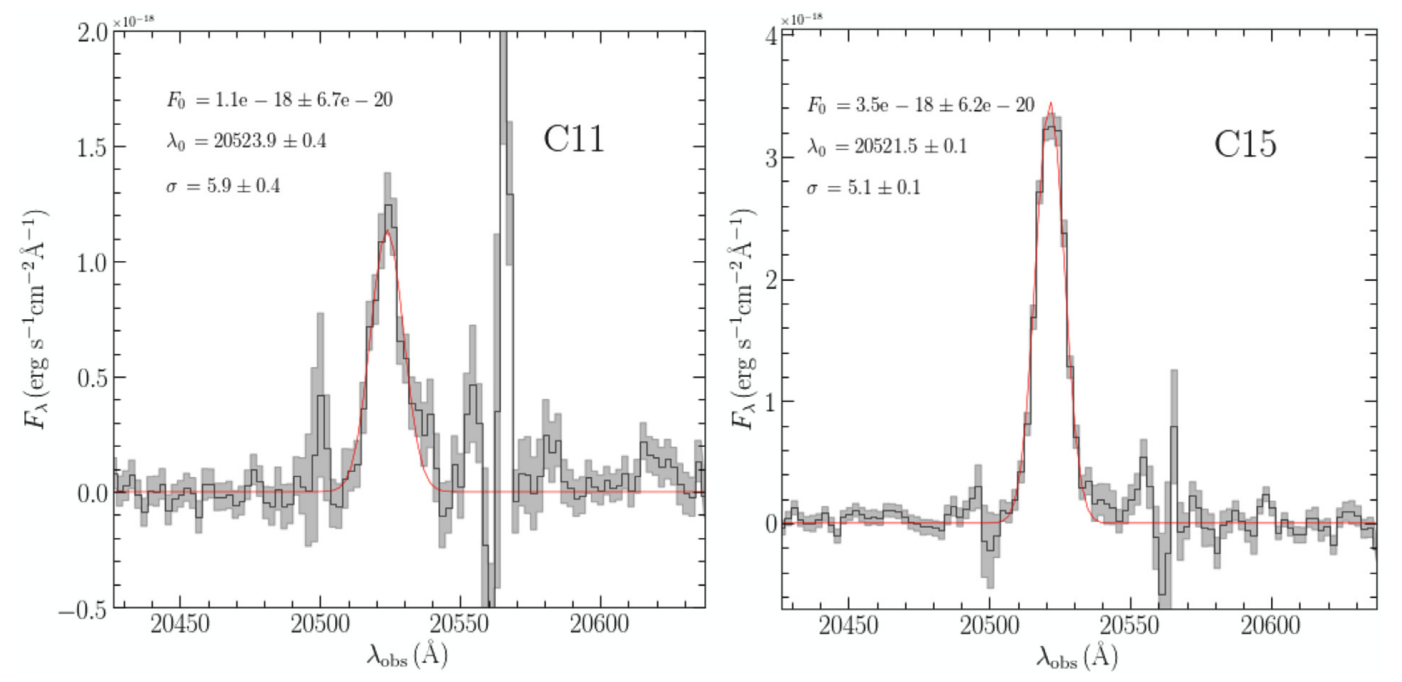

Figure A1. Single Gaussian fits to the observed [O III] profiles of two LBGs, C11 and C15. The [O III] emission is spatially integrated over the ranges indicated by black solid arrows in Fig. 3, which include all the significant [O III] emission of $\mathrm{C} 11$ and $\mathrm{C} 15$. The observed fluxes (shown in black) with $1 \sigma$ uncertainties (shaded in grey), the best-fitting model (shown in red) and best-fitting parameters (with $1 \sigma$ uncertainties) are shown in each panel. Note that skylines are present near the $[\mathrm{O} \mathrm{III}]$ lines, as indicated by large flux uncertainties.

\section{APPENDIX B: DERIVATION OF THE CRITICAL CLUMP COVERING FRACTION}

Here, we analytically derive the critical clump covering fraction, $f_{\mathrm{cl} \text {, crit }}$, for a multiphase medium whose kinematics have been defined earlier in this work, that is, HI clumps with a constant outflow velocity $v_{\mathrm{cl}}$ and a velocity dispersion $\sigma_{\mathrm{cl}}$, and an ICM with a constant outflow velocity $v_{\text {ICM. }}$. The optical depth of the H I in the ICM at the Ly $\alpha$ intrinsic frequency is approximated as $\tau_{0 \text {, ICM }}$, that is, the optical depth at the line centre. ${ }^{19}$

The large widths of the observed spectra imply that photons escape in a single long flight - after they have been scattered off the surface of a fast moving clump (Gronke et al. 2017). It follows that $f_{\text {cl, crit }}$ is given by the condition that on average one clump interacts with a photon at its intrinsic frequency.

The width in velocity space of each clump, $\tilde{v}$, is determined by:

$\tau_{\mathrm{cl}}( \pm \tilde{v} / 2)=\frac{4}{3} N_{\mathrm{HI}, \mathrm{cl}} \sigma_{\mathrm{HI}}( \pm \tilde{v} / 2)=1$

where $\tau_{\mathrm{cl}}$ is the optical depth of the clump, $N_{\mathrm{HI}, \text { cl }}$ is the $\mathrm{HI}$ column density of the clump, and $\sigma_{\mathrm{HI}}$ is the Ly $\alpha$ cross-section of the clump. The factor $4 / 3$ is simply due to the spherical geometry of the clump. Using the core approximation of the Ly $\alpha$ crosssection $\left(\sigma_{\mathrm{cl}}(v) \propto \exp \left(-v^{2} / v_{\mathrm{th}}^{2}\right)\right)$, the solution to equation (B1) can be explicitly written as $\tilde{v}=2 v_{\text {th }} \sqrt{\ln \tau_{0, \mathrm{cl}}}$, where $\tau_{0, \mathrm{cl}} \equiv \tau_{\mathrm{cl}}(v=0)$, and $v_{\text {th }}$ is the thermal velocity dispersion of $\mathrm{HI}$ within the clumps. For the H I column density and temperature of the clumps used in this work, $\tilde{v} \simeq 78 \mathrm{~km} \mathrm{~s}^{-1}$.

Under the assumption that all photons are injected at the Ly $\alpha$ line centre (which is a reasonable approximation for our setup since the width of the intrinsic spectrum $\sigma_{\mathrm{i}} \ll \sigma_{\mathrm{cl}}$ ), the average number of clumps per line of sight that intersect with $v=0$ in velocity space is:

$$
\tilde{f}_{\mathrm{cl}}=f_{\mathrm{cl}} \int_{-\tilde{v}}^{\tilde{v}} \mathcal{N}\left(v, \mu=v_{\mathrm{cl}, \|}, \sigma=\sigma_{\mathrm{cl}}\right) \mathrm{d} v
$$

where $\mathcal{N}$ denotes the normal distribution assumed for the velocity distribution of the clumps. ${ }^{20}$ Here,$v_{\mathrm{cl}, \text {,I }}$ is a component of $v_{\mathrm{cl}}$ that is parallel to the trajectories of photons. The reason for considering $v_{\mathrm{cl}, \text {, }}$ rather than $v_{\mathrm{cl}}$ is explained below.

Given the considerations above, demanding $\tilde{f}_{\mathrm{cl}}=1$ yields the critical number of clumps per line of sight (i.e. the clump covering fraction):

$$
f_{\mathrm{cl}, \mathrm{crit}}=\frac{2}{\operatorname{erf}\left(\frac{-v_{\mathrm{cl}, \|}+2 v_{\mathrm{th}} \sqrt{\ln \tau_{0, \mathrm{cl}}}}{\sqrt{2} \sigma_{\mathrm{cl}}}\right)+\operatorname{erf}\left(\frac{v_{\mathrm{cl}, \|}+2 v_{\mathrm{th}} \sqrt{\ln \tau_{0, \mathrm{cl}}}}{\sqrt{2} \sigma_{\mathrm{cl}}}\right)} .
$$

where $\operatorname{erf}(x)$ is the Gauss error function.

Note that this equation is a generalization of equation (12) in Gronke et al. (2017), where the radial velocity distribution of the clumps is approximated as a top-hat profile. It converges to that equation when $v_{\mathrm{cl}, ~ ॥} \ll \sigma_{\mathrm{cl}}$. For $v_{\mathrm{cl}, ~ ॥} \gg \sigma_{\mathrm{cl}}$, this equation yields very large values of $f_{\mathrm{cl} \text {, crit }}$.

Here, we discuss $v_{\mathrm{cl} \text {, „I }}$ for two different cases: (1) if $\tau_{0 \text {, ICM }} \ll 1$, initially the photons do not interact with the ICM. Therefore, $v_{\mathrm{cl}, \text {, }} \approx$ $v_{\mathrm{cl}} ;$ (2) if $\tau_{0 \text {, ICM }} \sim 1$ (up to a few), the photons can interact with the ICM prior to the clumps, and thus are likely to scatter orthogonally to the clump bulk outflow. Therefore, $v_{\mathrm{cl}, ~} \approx 0$ (with details depending on the exact value of $\tau_{0 \text {, ICM }}$ and the clump distribution). We do not consider $\tau_{0, \text { ICM }} \gg 1$ cases, where the multiphase, clumpy medium converges to a homogeneous medium that would fail to reproduce the observed spectra presented in this work (cf. discussion in Section 4).

\footnotetext{
${ }^{19}$ Technically speaking, the optical depth of the H I in the ICM is expressed as $\int \mathrm{d} v f_{\mathrm{i}}(v) \tau_{\mathrm{ICM}}(v)$ where $f_{\mathrm{i}}(v)$ is the normalized intrinsic spectrum as a function of velocity. As our intrinsic spectrum is very narrow, this approximation holds.
} 

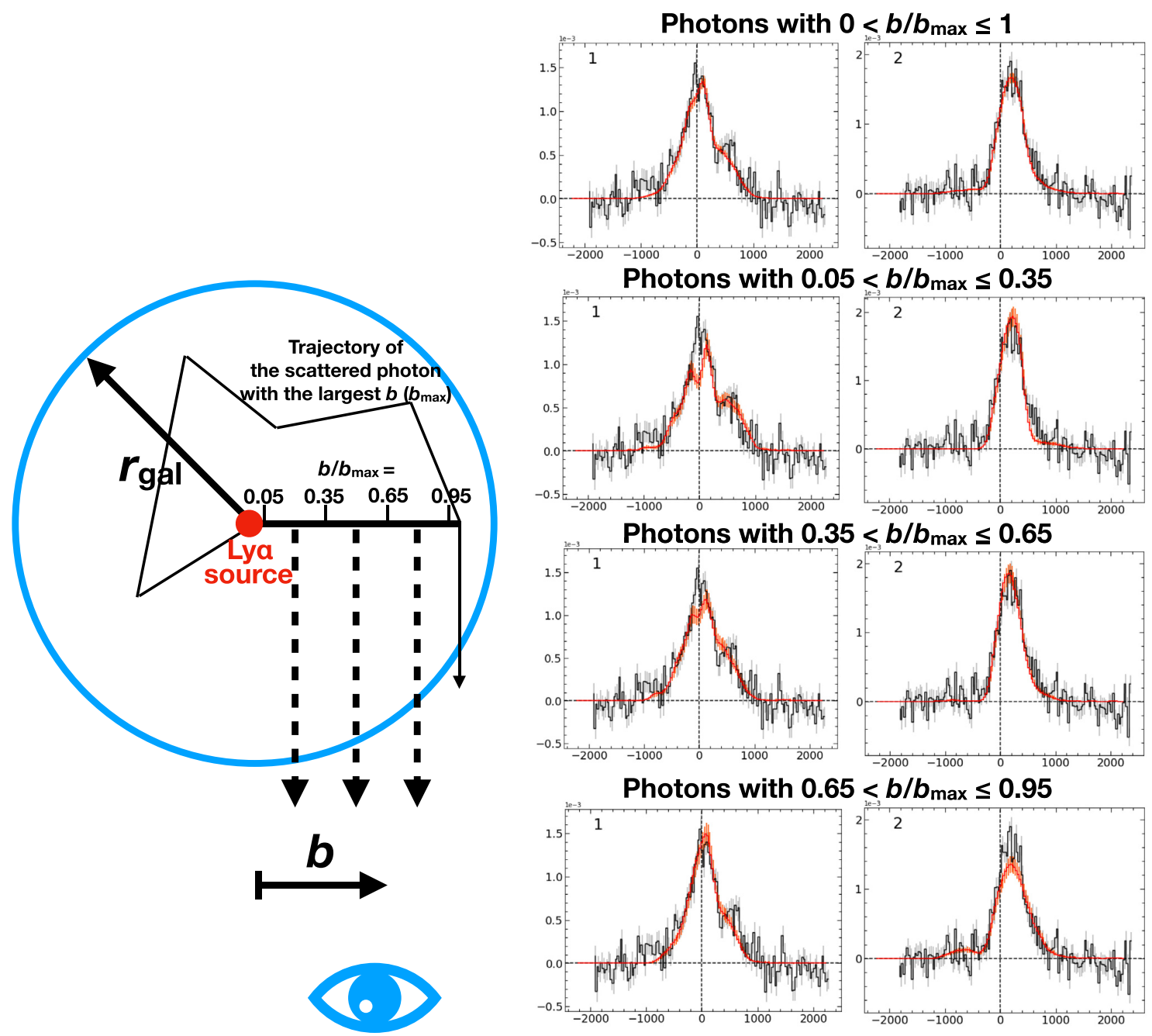

Photons with $0.05<b / b_{\max } \leq 0.35$

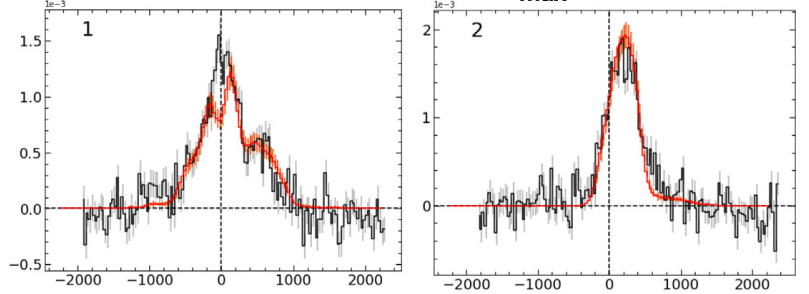

Photons with $0.35<b / b_{\max } \leq 0.65$

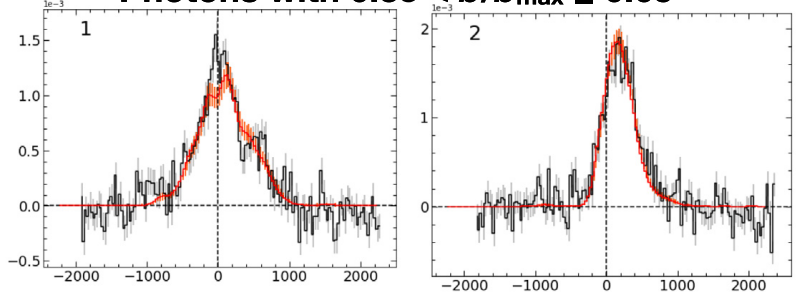

Photons with $0.65<b / b_{\max } \leq 0.95$

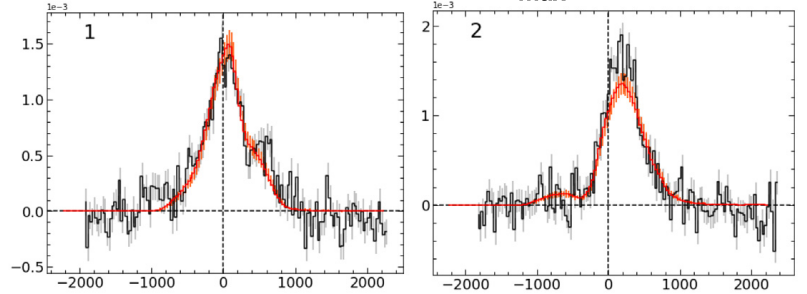

Figure C1. Justification of using spatially integrated models to fit the spatially resolved Ly $\alpha$ profiles. Left: configuration of the multiphase, clumpy model and the way we construct our photon bins for the observer. Right: comparison of integrated models and binned models for spectra 1 and 2. Assuming the largest impact parameter of all the photons is $b_{\max }$, we made three photon bins within the $2 \sigma$ range $\left(0.05<b / b_{\max } \leq 0.35,0.35<b / b_{\max } \leq 0.65\right.$, and $0.65<b / b_{\max }$ $\leq 0.95$ ) and constructed three binned model spectra with these photon bins, respectively. It can be seen the binned models are qualitatively very similar to the integrated model.

\section{APPENDIX C: JUSTIFICATION OF USING SPATIALLY INTEGRATED MODELS TO FIT THE SPATIALLY RESOLVED LY $\alpha$ PROFILES}

In this work, we have fitted spatially resolved Ly $\alpha$ profiles with spatially integrated models, which are derived from Ly $\alpha$ photons from the entire scattering region. Here, we emphasize that qualitatively, these spatially integrated models are very similar to the binned models, which are derived from Ly $\alpha$ photons within a certain impact parameter range.

We illustrate this in Fig. C1. With spectra 1 and 2 as two examples, we binned all the scattered photons according to their impact parameters, $b$ (the projected distance to the simulation centre perpendicular to the line of sight). Assuming the largest impact parameter of all the photons is $b_{\max }$, we made three photon bins within the $2 \sigma$ range $\left(0.05<b / b_{\max } \leq 0.35,0.35<b / b_{\max } \leq 0.65\right.$, and $0.65<b / b_{\max } \leq 0.95$ ) and constructed three binned model spectra with these photon bins respectively. It can be seen the binned models are qualitatively very similar to the integrated model. Therefore, it is approximately correct to model the spatially resolved profiles at positions away from the $\operatorname{Ly} \alpha$ emitting sources with spatially integrated models.

The aim of our modelling in this work is to roughly extract the velocities and densities of H I that the Ly $\alpha$ photons 'experience' in situ. In our following work, we plan to model the spatially resolved profiles in a more self-consistent way (e.g. modelling the spatially resolved Ly $\alpha$ profiles with a common scattering medium).

\section{APPENDIX D: POSTERIOR PROBABILITY DISTRIBUTIONS DERIVED FROM NESTED SAMPLING}

Here, we present the joint and marginal posterior probability distributions of the multiphase clumpy model parameters for all 11 representative $\operatorname{Ly} \alpha$ spectra derived from nested sampling. For each spectrum, we also show the best fit, the observed Ly $\alpha$ profile and five model spectrum samples from nested sampling. 


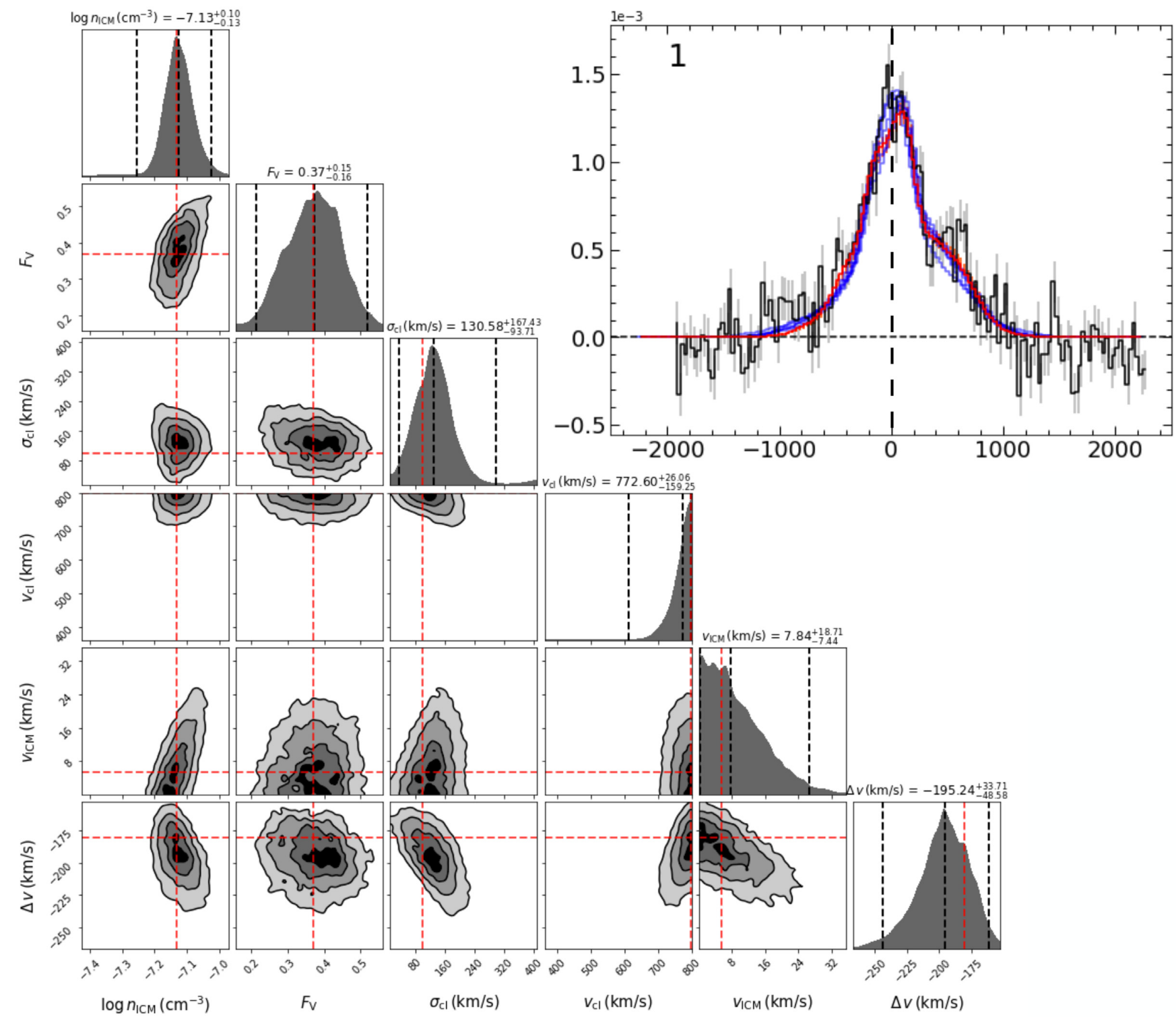

Figure D1. Joint and marginal posterior probability distributions of the multiphase clumpy model parameters for all 11 representative Ly $\alpha$ spectra derived from nested sampling. The vertical black dashed lines indicate the [ 2.5 per cent, 50 per cent, 97.5 per cent] quantiles (i.e. $2 \sigma$ confidence intervals). The vertical red dashed lines indicate the locations of the maximum posterior probability. The upper right panels show the best fits (red, with orange $1 \sigma$ Poisson errors), the observed Ly $\alpha$ profiles (black, with grey $1 \sigma$ error bars) and five model spectrum samples from nested sampling (blue) in the same way as Fig. 5. 


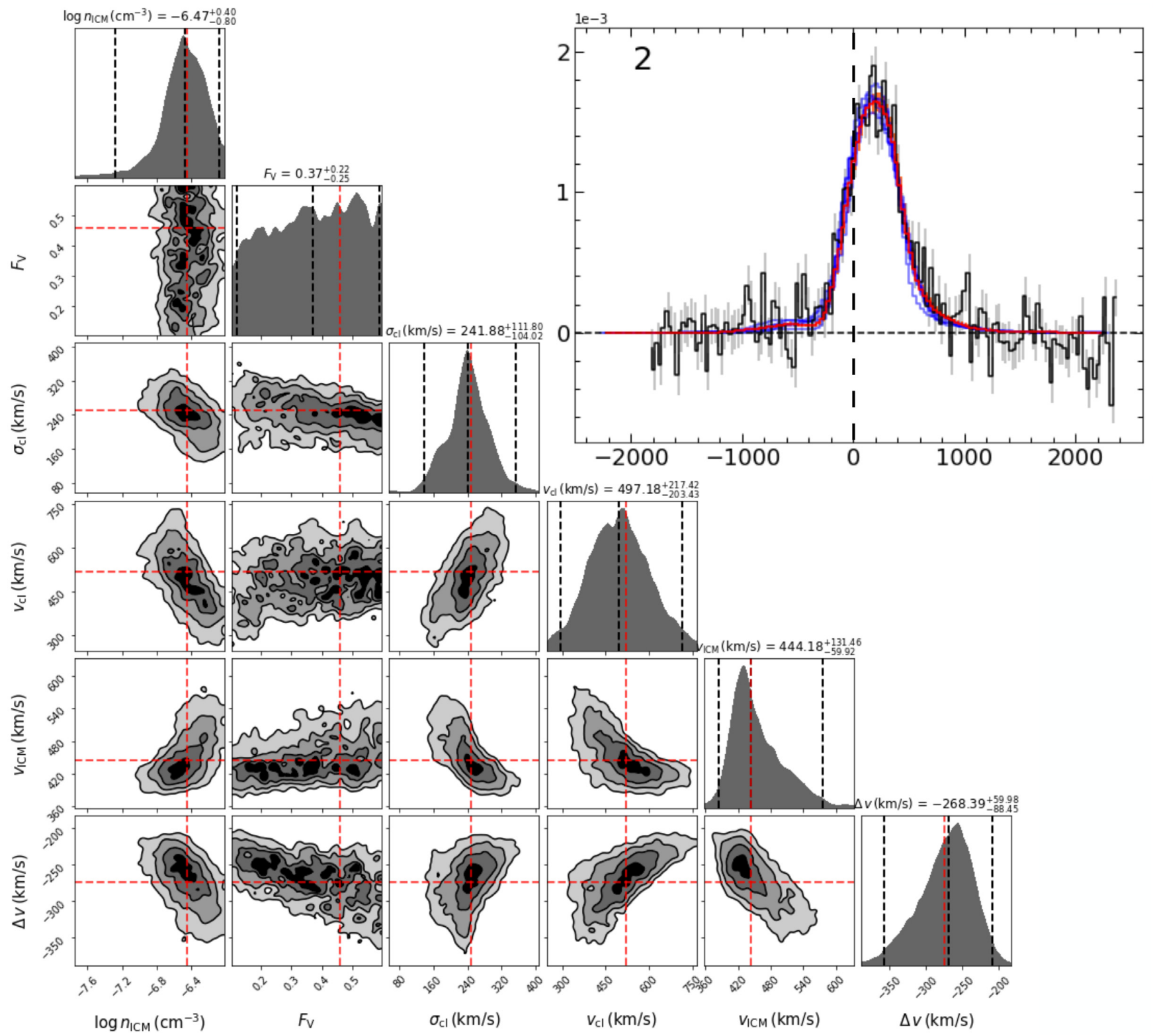

Figure D1. continued. 


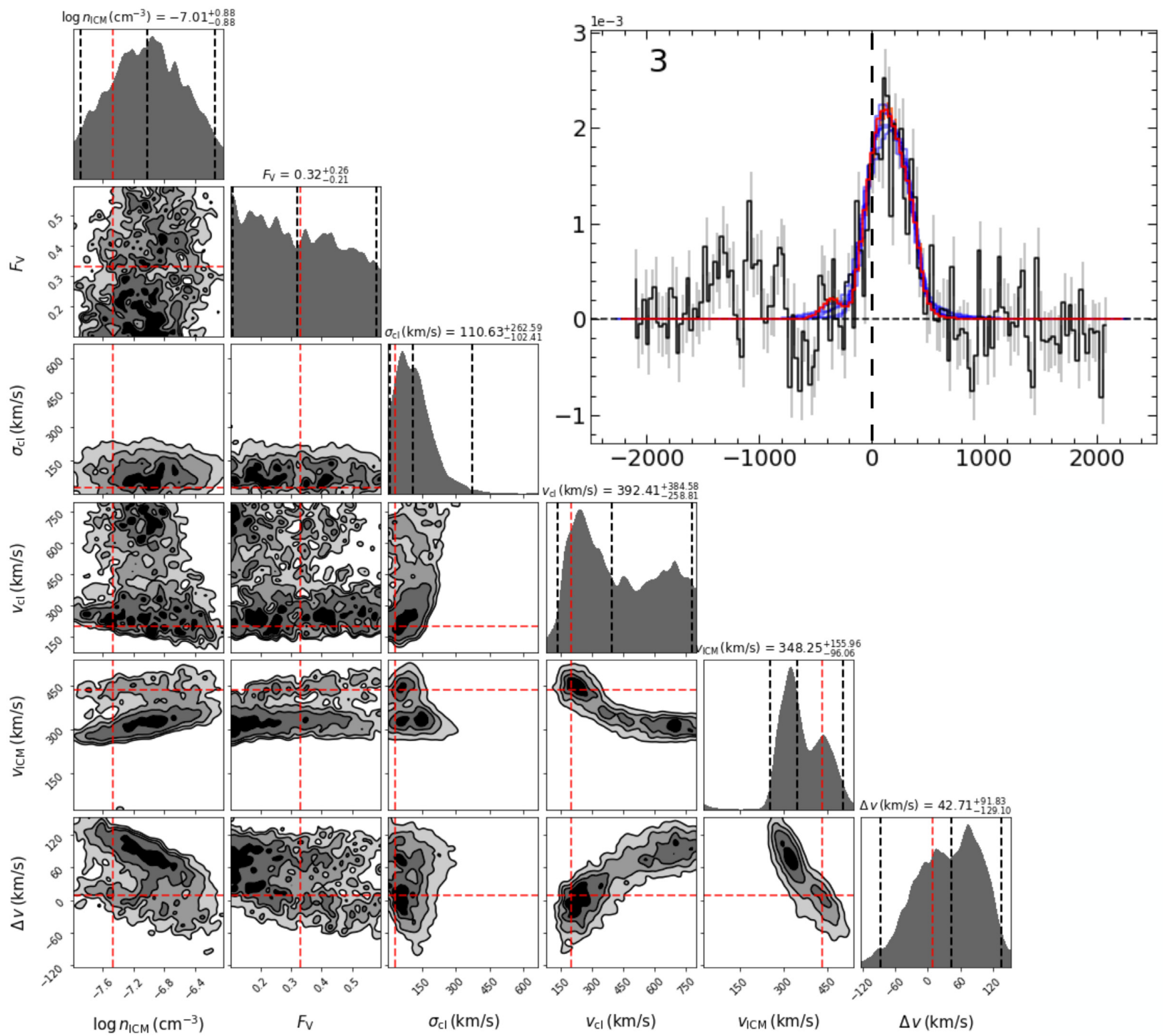

Figure D1. continued.

This paper has been typeset from a $\mathrm{T}_{\mathrm{E}} \mathrm{X} / \mathrm{LT} \mathrm{T} \mathrm{X}$ file prepared by the author. 